\title{
LIVE, OPTICAL EXPERIMENTAL NEUROIMAGING REVEALS \\ VARIATIONS OF THE CEREBRAL BLOOD FLOW RESPONSE TO ISCHEMIC SPREADING DEPOLARIZATION
}

\author{
Zsófia Bere MD \\ $\mathrm{PhD}$ Thesis
}

University of Szeged, Department of Medical Physics and Informatics

PhD Program:

Theoretical Medicine

Program director: Prof. Gábor Jancsó D.Sc.

Supervisor: Eszter Farkas PhD

Szeged, 2016 


\section{Publications related to the $\mathrm{PhD}$ Thesis}

I. Bere Z, Obrenovitch TP, Bari F, Farkas E. Ischemia-induced depolarizations and associated hemodynamic responses in incomplete global forebrain ischemia in rats. Neuroscience. $2014 \mathrm{Feb} 28 ; 260: 217-26$. doi:

10.1016/j.neuroscience.2013.12.032. Epub 2013 Dec 21. IF:3.32

II. Bere Z, Obrenovitch TP, Kozák G, Bari F, Farkas E. Imaging reveals the focal area of spreading depolarizations and a variety of hemodynamic responses in a rat microembolic stroke model. J Cereb Blood Flow Metab. 2014 Jul 30. doi: 10.1038/jcbfm.2014.136. IF:5.33 


\begin{tabular}{|c|c|}
\hline $\mathrm{aCSF}$ & arteficial cerebrospinal fluid \\
\hline $\mathrm{AD}$ & anoxic depolarization \\
\hline AOI & area of interest \\
\hline CBV & cerebral blood volume \\
\hline $\mathrm{CCA}$ & common carotid artery \\
\hline DC & direct current \\
\hline ECA & external carotid artery \\
\hline ECoG & electrocorticography \\
\hline GABA & $\gamma$-aminobutyric acid \\
\hline $\mathrm{Hb}$ & hemoglobin \\
\hline ICA & internal carotid artery \\
\hline IOS & intrinsic optical signal \\
\hline LED & light emitting diode \\
\hline LSC & laser speckle contrast \\
\hline MAP & mean arterial pressure \\
\hline MCAO & middle cerebral artery occlusion \\
\hline $\mathrm{Na} / \mathrm{K}$ ATPase & ATP consuming sodium pump \\
\hline NO & nitrogen monoxid \\
\hline $\mathrm{SD}$ & spreading depolarization \\
\hline TTC & 2,3,5-triphenyltetrazolium chloride \\
\hline VS & voltage sensitive \\
\hline $2 \mathrm{VO}$ & bilateral occlusion \\
\hline
\end{tabular}




\section{Table of content}

1. Introduction 1

1.1. Regional cerebral blood flow response to spreading depolarisation- 2 neurovascular coupling

1.2. Patophysiological impact of spreading depolarization

1.3. Assessment of ischemia induced spreading depolarization and neurovascular coupling

2. Aims

3. Materials and methods $\quad 8$

$\begin{array}{ll}\text { 3.1. Surgical procedures } & 9\end{array}$

3.1.1. Preparations for the induction of incomplete global forebrain ischemia 9

3.1.2. Preparations for the induction of microembolic stroke 9

3.1.3. Closed cranial window preparation $\quad 9$

3.2. Experimental protocol 10

3.2.1. Incomplete global forebrain ischemia model 10

3.2.2. Microembolic stroke model 11

3.3. Multi-modal imaging 11

3.3.1. Imaging of cellular membrane potential changes 11

3.3.2. Imaging of cerebral blood flow changes 12

3.3.3. Imaging changes in cerebral blood volume and hemoglobin saturation 12

3.3.4. Pial arteriolar diameter 13

3.4. Protocol for image analysis 14

$\begin{array}{ll}\text { 3.5. Histology in microsphere model } & 14\end{array}$

4. Results 15

4.1. Changes int he aquired variables with ischemia induction 15 
4.1.2. Multifocal ischemia

4.2. Characterization of spreading depolarizations and associated hemodynamic responses

4.2.1. Global forebrain ischemia

4.2.2. Multifocal ischemia

4.2.2.1. Focal events and associated hemodynamic changes

4.2.2.2. Features of propagating spreading depolarizations and the associated cerebral blood flow response

5. Discussion

5.1. The general impact of ischemia induction on mean arterial blood pressure and cerebral blood flow

5.1.1. Global forebrain ischemia model

5.1.2. Multifocal cerebral ischemia model

5.2. Threshold of ischemia induced depolarization waves

5.3. The focal area of spreading depolarizations

5.4. Kinetics of spreading depolarization and the associated cerebral blood flow response

5.5. Unique features of spreading depolarizations over their course of propagation

6. Conclusions and future perspectives

7. References 



\section{Introduction}

Stroke is the third most common cause of death in Hungary and Europe, coming only after heart disease and cancer. Much of the primary damage in the acute phase of ischemic stroke may prove irreversible despite prompt intervention, yet the management of secondary pathophysiological processes is more feasible and of fundamental importance to improve the prospect of successful recovery.

The neurological deficit caused by stroke, thus, obviously depends upon the severity and nature of the initial injury, but also upon secondary and progressive deleterious events, such as spreading depolarization (SD). Research on SD considerably accelerated in the last fifteen years due to the highly significant observation that spontaneous SDs occur consistently and repeatedly in the injured human brain, and contribute to lesion progression. This justifies research efforts to understand the pathophysiological role of $\mathrm{SD}$ in ischemic brain injury.

In 1944, A.P. Leao described that direct electrical stimulus of cerebral cortex causes transitional suppression of spontaneous cortical activity seen as transient depression of the electrocorticogram $(\mathrm{ECoG})$ [1]. Using a series of electrodes positioned at increasing distances from the site of electrical stimulation, the phenomenon was detected first at the recording site nearest the stimulus, and spread with $3-6 \mathrm{~mm} / \mathrm{min}$ to the more distant electrodes. The recovery of cortical activity started within seconds close to the site of electrical stimulation and appeared with a similar delay at subsequent electrodes (Fig. 1). Based on these observations, the phenomenon was named as "spreading depression of cortical activity".

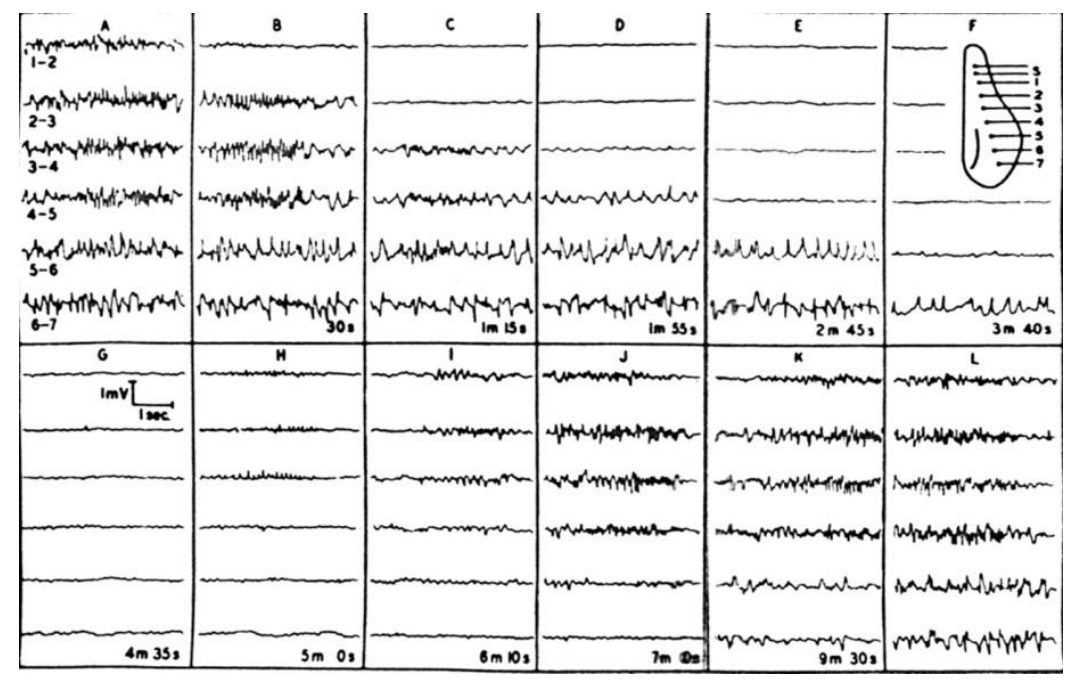

Figure 1: Leao's original illustration of spreading depression. The traces correspond to electrocorticogram channels, the signals acquired at the surface of the brain of a rabbit. The time elapsed after electric stimulation is displayed at the bottom of each panel. The inset (F) shows the position of the electrodes: s, stimulation; $1-$ 7 , recording electrodes paired as indicated in $(\mathrm{A})$. 
Subsequent electrophysiological studies that recorded direct current (DC) potential demonstrated, that suppression of the ECoG coincided with a near-complete, sustained depolarization of a critical population of cortical neurons (Fig. 2).

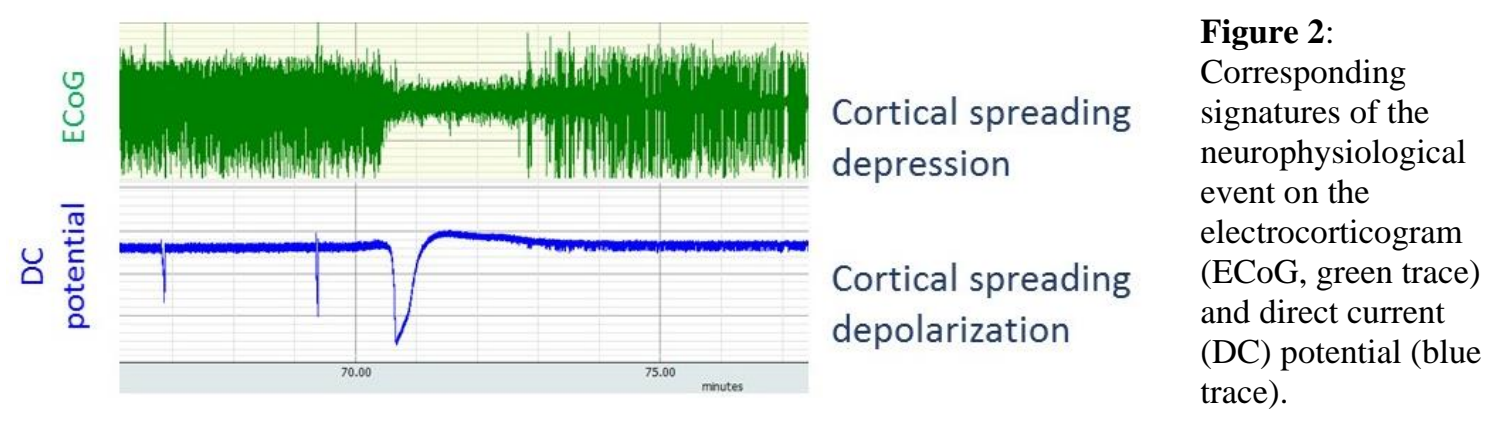

This feature of the events has served to unify the terminology and define propagating depolarization events (in intact, ischemic or anoxic tissue) as spreading depolarization [2, 3] The depolarization is caused by the breakdown of ion equilibrium across neuronal membranes [4] during the depolarization phase of SD, there is an intense efflux of $\mathrm{K}^{+}$to the extracellular space, parallel with considerable influx of $\mathrm{Na}^{+}[5,6]$. As SD propagates across the cortex, considerable osmotic changes follow the ionic redistribution, which manifests in swelling of neurons and dendritic spines [7, 8]. Due to sustained depolarization, neuronal membrane channels producing action potential are inactivated; therefore additional stimuli cannot evoke action potential. This forms the basis of electrical silence indicative of SD on ECoG signal [1]. The complex mechanisms of SD propagation are still a target of investigation. Instead of relying on synaptic connections conveying depolarization to sites distant to neuronal cell bodies, SD propagates to adjacent neuron populations, possibly via direct gap junctions [9] Also, the mass release of excitatory neurotransmitters such as glutamate, acetylcholine and $\gamma$-aminobutyric acid (GABA) might serve volume transmission $[6,10]$.

\subsection{Regional cerebral blood flow response to spreading depolarization- neurovascular coupling}

Since restoration of transmembrane potential after SD relies on ATP-dependent ion pumps, the obvious metabolic demand requires rapid increases in oxygen and glucose delivery, which is mediated by neurovascular coupling. Neurovascular coupling is a vital feed-forward control mechanism, which adjusts local cerebral blood flow (CBF) to the energy requirements of activated neurons [11]. The resulting functional hyperemia increases the delivery of oxygen and essential nutrients, affords effective washout of 
waste-products, and maintains an optimal homeostatic balance in the local neural microenvironment. Neurovascular coupling is based on an orchestrated, tightly controlled intercellular communication between activated neural elements such as neurons and astrocytes, and vascular compartments including endothelial cells, pericytes and smooth muscle cells. The cellular mechanisms underlying neurovascular coupling are multiple: in addition to the neuronal production of the vasodilator nitric oxide (NO) [12], the role of astrocytes in neurovascular coupling is becoming increasingly clearer. Astrocytes embracing the synaptic cleft and having multiple endfeet opposed to microvascular smooth muscle cells - are ideally situated to function as relay cells in neurovascular communication. Recent discoveries provided strong evidence that astrocytes can control local cerebral blood flow through $\mathrm{Ca}^{2+}$-dependent production and release of metabolites of arachidonic acid (e.g. epoxygenase-derived epoxyeicosatrienoic acids, and cyclooxygenase-derived prostaglandins) [13]. SDs are coupled with typical changes in local CBF. In the rat - and most probably in human - the physiological pattern of the SDassociated CBF response includes four sequential components: (i) an initial, brief hypoperfusion; (ii) a marked, transient peak hyperemia; (iii) a less obvious late hyperemia; and (iv) a sustained hypoperfusion also known as spreading oligemia or postSD oligemia[14]. The duration and magnitude of these four elements in the CBF response is variable, with the peak hyperemic component being the most conspicuous. The pronounced hyperemia often exceeds $100 \%$ with respect to baseline and outlasts SD for minutes [15]. After peak hyperemia, CBF stabilizes below baseline level and persists at oligemic values for 30-60 min. The different phases of the SD-related CBF response are mediated by a combination of extracellular ionic, neurotransmitter, and metabolic changes [14]. These include the increase of interstitial $\mathrm{K}^{+}$(staying below $20 \mathrm{mM}$ vasodilator, rising above $20 \mathrm{mM}$ vasoconstrictor), variation in the release of nitric oxide or prostaglandins, or the possible modulation of adenosine receptors [14]. These interactions are rather complex and must depend on the metabolic status of the tissue, therefore the discrimination of individual factors remains a challenge.

\subsection{Patophysiological impact of spreading depolarization}

For decades, SD was speculated to develop only under experimental conditions, because no clinical evidence supported the occurrence of SD in the human brain. Although Leao specifically linked SD to migraine aura and cerebral ischemia [16], the 
technical developments of diagnostic tools enabled the detection of SD in patients only decades later. First, the introduction of PET revealed the CBF response to SD in patients of migraine with aura [15]. In 2002, a groundbreaking study positioning surface ECoG strips on the cortex of postoperative neurosurgical patients demonstrated that SDs occur and propagate in the injured human neocortex [17]. This revealing finding initiated a subsequent line of influential studies to investigate the pathophysiological relevance of SD in patients of malignant stroke, subarachnoid hemorrhage and traumatic brain injury (cf. www.cosbid.org [17-23]).

SDs have long been suspected to aggravate ischemic injury. In particular, SDs have been considered to recruit electrically silent but viable penumbra tissue into the nonviable core region - thereby expanding the injury [24-26]. This notion was supported by the coincidence of SD evolution and infarct maturation demonstrated by a linear correlation between the total number or cumulative duration of recurrent SDs and the infarct volume after middle cerebral artery occlusion (MCAO) in rats [27, 28]. In subarachnoid hemorrhage patients, delayed ischemic neurological deficit corresponded with a sequence of recurrent SDs [20]. After traumatic brain injury, SDs of long duration and frequent recurrence in temporal clusters, in association with isoelectricity of the ECoG or periodic epileptiform discharges predicted unfavorable outcome [22, 23]. The relationship between SD occurrence and injury progression appears causal, because $\mathrm{KCl}$ induced SDs invading the penumbra from remote sites increased infarct volumes in experimental stroke [24, 29].

Under ischemic conditions, spontaneous SDs occur within minutes. However, the duration of depolarization may vary greatly, dependent on the metabolic status of the tissue. The restoration of transmembrane potential i.e. repolarization is achieved by the ATP consuming sodium pump ( $\mathrm{Na} / \mathrm{K}$ ATPase). If oxygen and nutrient supply is undisturbed, the depolarization is short transient, and repolarization is complete within a minute [30]. In case a mismatch between energy demand and supply evolves (i.e. due to perfusion deficit), the efficacy of the sodium pump will decrease due to the restricted availability of ATP, and repolarization will be delayed, coincident with a persistent but reversible depression of cortical activity [20, 25]. This is typical of the ischemic penumbra [31]. Finally, severe shortage of oxygen and energy substrates as it occurs after cardiac arrest or in the core region of focal ischemia impedes reporlarization, and leads to terminal depolarization. The ability of neurons to repolarize indicates viability, while terminal depolarization is a clear sign of neuronal death. Taken together, the duration of 
depolarization can be indicative of the metabolic crisis, and is attributed a predictive value as to injury outcome $[3,30,32]$.

The mechanisms behind the injurious potential of SD are still being investigated. At present, the deficiency of the SD-associated $\mathrm{CBF}$ response is accepted to mediate neurodegeneration, because the insufficient $\mathrm{CBF}$ response sustains the state of depolarization and delays repolarization by depriving the tissue at risk of essential nutrients required to maintain ionic balance across neuronal cell membranes [14, 32].

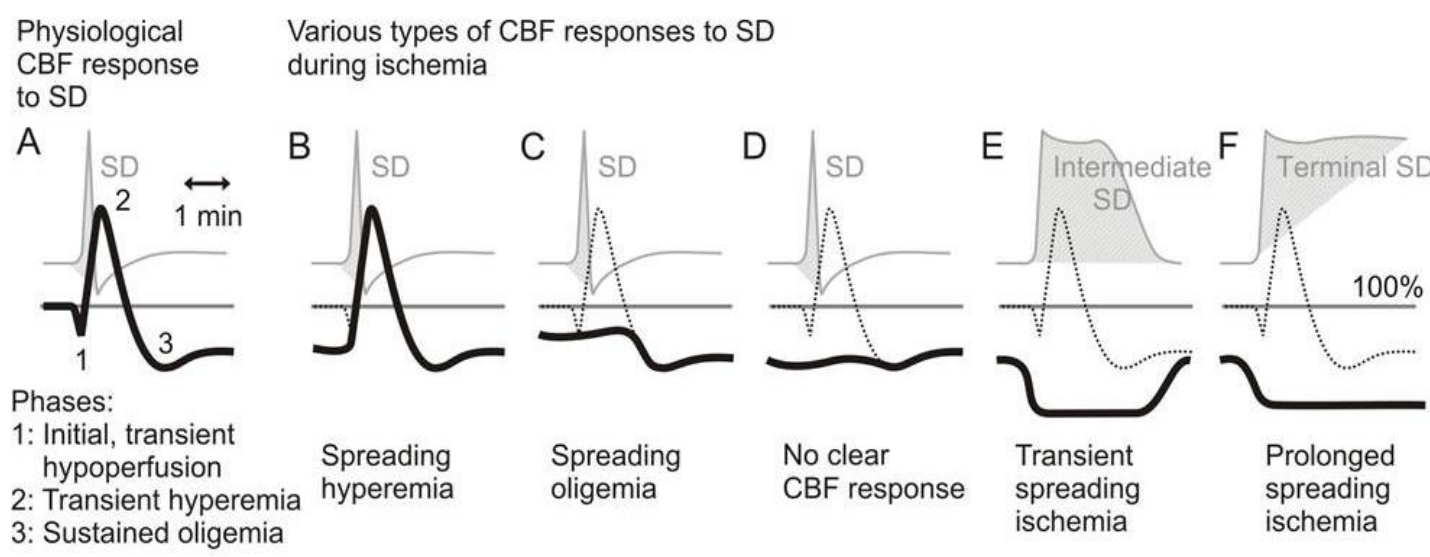

Figure 3: Overview of various conceptual cerebral blood flow $(\mathrm{CBF})$ responses to spreading depolarization (SD) in the intact cerebral cortex (A), and during ischemia (B-F). Solid, black lines depict theoretical CBF responses based on our observations. Dotted lines in panels B-F indicate the physiological CBF response to $\mathrm{SD}$, for comparison with those under ischemia. Gray lines and shaded areas stand for ideal SD (negative deflection upwards): short transient in panels $\mathrm{A}-\mathrm{D}$, intermediate in panel $\mathrm{E}$, and terminal in panel $\mathrm{F}$. Baseline CBF (100\%) is given as a horizontal line [34].

In contrast to the hyperemic response related to SD in healthy tissue, impaired neurovascular coupling under ischemic conditions induces severe vasoconstriction, and leads to marked, prolonged hypoperfusion, which spreads together with SD. In the ischemic brain the CBF response to SD may undergo a gradual transformation to uncover ruling vasoconstrictive elements (Fig. 3). The most important parameter in the spectrum from normal hyperemic responses to inverse responses is the growing presence and increasing duration of the initial hypoperfusion [20,33].

The perfusion deficit in the ischemic tissue is aggravated by spreading ischemia which worsens the mismatch between energy demand and supply in the tissue, markedly delays the recovery from SD, and shifts prolonged SD to terminal depolarization [35]. The normal hemodynamic response to SD is achieved by a fine balance between constrictive and dilator vasoregulatory mechanisms [14]. Under physiological condition, vasoconstriction caused by increased extracellular $\mathrm{K}+$ concentration is surmounted by 
vasodilation mediated by decreased tissue $\mathrm{pH}$ and $\mathrm{NO}$ release [36, 37]. Ischemia alters the baseline tone of arteries: shortly after the occlusion of a cerebral vessel, vasodilation evolves in order to maintain CBF against decreasing perfusion pressure. This leads to maximal vasodilation and exhausts microvascular reserve capacity. Days after the primary injury, vasospasm may develop, which is implicated in delayed cerebral ischemia after subarachnoid hemorrhage [38]. Against these general cerebrovascular changes, SDcoupled vasoconstriction may prevail, because counteracting vasodilators that would normally balance out vasoconstriction are not available. As such, when NO availability is decreased (for example NO is scavenged by hemoglobin), together with an elevated concentration of extracellular $\mathrm{K}^{+}$, the net vasodilator effect cannot overcome $\mathrm{K}^{+}$induced vasoconstriction [39]. Moreover, increased basal extracellular $\mathrm{K}^{+}$concentration contributes to increased calcium $\left(\mathrm{Ca}^{2+}\right)$ storage in astrocytes and vascular smooth muscle, and during depolarization, massive $\mathrm{Ca}^{2+}$ release enhance vasoconstriction [40]. The result is a vicious cycle: ischemia favors the initiation of recurrent spreading depolarizations, some of which are coupled with spreading ischemia. Such events will deepen the perfusion deficit in a stepwise fashion, which will give rise to further depolarization events [41, 42]. Finally prolonged depolarization with spreading ischemia extends cortical necrosis, and enlarges the ischemic core [43, 44].

\subsection{Assessment of ischemia induced spreading depolarization and neurovascular coupling}

The characterization of SD waves traditionally relies on the recording of extracellular DC potential from the cerebral cortex, which is an accurate and suitable approach, but lacks spatiotemporal resolution. Our group has developed and validated a method for direct, live imaging of $\mathrm{K}^{+}$-induced SD in a rat closed cranial window preparation, using a voltage-sensitive (VS) dye [45]. VS dyes are chemicals that bind to cellular membranes and whose fluorescence increases with reduced cellular transmembrane potential [46]. Our group has demonstrated that the SD-associated changes of VS dye fluorescence with time are equivalent to the electrical signature of SD (i.e. DC potential shift) [45]. The two-dimensional spatial resolution feature of VS dye imaging is uniquely appropriate to monitor the site of initiation of SDs, and their subsequent route of propagation, which cannot be predicted in the ischemic cortex. Another advantage of the imaging approach is that, once SD elicitation has been identified on relevant captured images, small areas of 
interest can be selected, and used as probes to extract the kinetics of changes in VS dye fluorescence within the whole image sequence. This quantitative feature of the technique (i.e. determination of SD kinetics at any chosen cortical location within the field of view) is highly pertinent for our objectives, given that we will deal with events elicited in regions subjected to various levels of ischemia severity, and where individual ischemia intensity may evolve with the maturation and progression of the lesion.

Next, the VS dye method was extended by coupling it to laser speckle contrast (LSC) blood flow imaging [47]. LSC imaging provides information on the velocity of blood cells, and thereby data on relative blood flow changes. This is now a well validated approach for the imaging of local CBF changes with high temporal and spatial resolution [48]. The VS dye/ LSC imaging combination has the capability to provide data sets that are perfectly matched, in both space and time, for the two variables. By itself, LSC imaging enables the monitoring of the evolution and degree of ischemia, and following the $\mathrm{CBF}$ dynamics at various cortical sites after ischemia onset. In association with SD imaging (i.e. VS dye method), LSC can identify the potentially severely affected and spared cortical regions, and correlate SD features with local ischemia severity and its evolution.

Finally, the multimodal imaging approach has been completed by the analysis of intrinsic optical signals (IOS) [49]. IOS evoked with 540 to $550 \mathrm{~nm}$ green light illumination allows the estimation of local cerebral blood volume (CBV): decreasing green IOS intensity (i.e., decreasing intensity of reflected light) is accepted to correlate with increasing CBV. Finally, IOS under 620 to $640 \mathrm{~nm}$ red light illumination is indicative of the oxygen saturation of hemoglobin: decreasing red IOS intensity represents decreasing hemoglobin saturation; in case hemoglobin is totally desaturated, red IOS intensity changes follow that of CBV [49].

The rat MCAO model for stroke is a widely used method to study the pathogenesis of focal brain infarcts [50]. Moreover, SDs are readily generated after MCAO, which renders the model suitable for the investigation of ischemia-induced SD [51]. As an alternative to MCAO, the microsphere-induced, multifocal stroke model in rats has been utilized to mimic the formation of small, permanent cerebral emboli and multiple infarct areas $[52,53]$. Such a multifocal stroke model appears to be more suitable to capture SD elicitation sites in the parietal cortex of the rat. The model is relevant for small embolic infarcts in patients, complications with cardiac or pulmonary arteriovenous shunts (e.g., cerebral emboli), and possibly multiinfarct dementia. Whether SDs occur in microsphere- 
induced, multifocal stroke in the rat brain has not been shown, but recurrent SDs were successfully evoked in the microembolized mouse brain [54].

SDs are generally considered to be initiated at sharply demarcated ischemic border zones in focal ischemia. However, it remains unclear whether depolarizations may also occur in non-terminal global ischemia, i.e. conditions in which no distinct ischemic core and penumbra can be delineated, and thereby no marked heterogeneous ischemia be anticipated. The investigation of depolarizations in global ischemia may be relevant to human conditions such as hypovolemic shock, cardiac arrest or complex cardiac surgery, which involve a risk of reduced $\mathrm{CBF}$ and related poor neurologic outcome.

\section{Aims}

Because the insufficiency of the CBF response to SD has been suspected to mediate SD-related injury, our major goal was to identify various types of hemodynamic responses to $\mathrm{SD}$ with relation to the severity of cerebral ischemia, and to determine a possible association between depolarization types and associated hemodynamic responses. Further objectives included the characterization of specific conditions that favor SD elicitation and propagation, and of patterns of SD propagation over cortical areas with various severity of ischemia.

\section{Materials and methods}

All animal procedures were approved by the Ethical Committee for Animal Care of the University of Szeged adhering to national regulation (guide- lines of the Scientific Committee of Animal Experimentation of the Hungarian Academy of Sciences, updated Law and Regulations on Animal Protection (40/2013. (II. 14.) of Hungary), following the EU Directive 2010/63/EU.

Inhalation anesthesia was performed on adult male Sprague-Dawley rats with 1.5-2.0\% halothane in $\mathrm{N}_{2} \mathrm{O}: \mathrm{O}_{2}(2: 1)$. Breathing was spontaneous throughout surgical preparation and subsequent data acquisition. Body temperature was kept between $37.1-37.4^{\circ} \mathrm{C}$ using a heating pad feedback-controlled by a rectal probe. To avoid overproduction of airway mucus, rats were pretreated with $0.05 \mathrm{ml}$ atropine intramuscularly. 


\subsection{Surgical procedures}

\subsubsection{Preparations for the induction of incomplete global forebrain ischemia}

Rats $(\mathrm{n}=13,300-450 \mathrm{~g})$ were anaesthetized. The left femoral vein and artery were cannulated for the monitoring of mean arterial pressure (MAP), and latter induction of hypovolemic hypotension. Midline cervical incision was made to expose the common carotid arteries (CCA). The vessels were gently separated from their sheaths and vagal nerves. Surgical sutures were loosely looped around the common carotid arteries for permanent bilateral occlusion (2VO) during image acquisition. The ends of surgical threads were guided out through small lateral incisions in the skin above the common carotid arteries, and the midline wound was closed.

\subsubsection{Preparations for the induction of microembolic stroke}

Rats ( $\mathrm{n}=13,300-450 \mathrm{~g})$ were anaesthetized. The right femoral artery was cannulated for the monitoring of MAP. Ventral cervical incision was made in the midline of the neck to expose the right CCA. The vessel was separated from the adjacent tissue. The external and internal carotid arteries (ECA, ICA) were also liberated. The ECA and the CCA were ligated proximally. A polyethylene tube $(1.0 \mathrm{~mm}$ OD x $30 \mathrm{~cm}$ Intravenous Cannula, Sims Portex, Smith Indrustries, Hythe, Kent, England) was inserted into the right CCA and was advanced gently into the ICA until it reached the base of the skull. The catheter was secured to the vessel with surgical sutures. The midline wound was closed.

\subsubsection{Closed cranial window preparation}

In both models, after the surgical steps above, animals were placed in a stereotactic frame in the prone position. A cranial window (approximately $4 \times 4 \mathrm{~mm}$ ) was prepared at the center of the right parietal bone by carefully thinning the bone with a high-precision electrical drill (Technobox 810, Bien-Air Dental SA, Bienne, Switzerland). A doughnut shape ring of methylmethacrylate resin (GC Unifast TRAD, GC Europe-GC EEOHungary, Hungary) was built around the craniotomy, incorporating a perfusion inlet and outlet, the inlet connected to a peristaltic pump. The chamber was filled with artificial cerebrospinal fluid (aCSF; mM concentrations: $126.6 \mathrm{NaCl}, 3 \mathrm{KCl}, 1.5 \mathrm{CaCl}_{2}, 1.2 \mathrm{MgCl}_{2}$, $24.5 \mathrm{NaHCO}_{3}^{-}, 6.7$ urea, 3.7 glucose, bubbled with $95 \% \mathrm{O}_{2}$ and $5 \% \mathrm{CO}_{2}$ to achieve a constant $\mathrm{pH}$ of 7.45 ), and the dura carefully dissected before sealing the cranial window 
with a glass coverslip, glued with a two-component-glue (UHU GmbH \& Co, KG; Bühl, Germany) to the dental cement ring. Unless otherwise specified, the closed chamber was continuously perfused with aCSF at a rate of $25 \mu \mathrm{L} / \mathrm{min}$. The pressure in the closed cranial window was kept near the physiologic intracranial pressure by securing the end of the perfusion outlet at $13 \mathrm{H}_{2} \mathrm{Ocm}$ above the animals' head.

\subsection{Experimental protocol}

In each experiment, the VS dye was dissolved in aCSF and circulated over the exposed cortex $(80 \mu \mathrm{l} / \mathrm{min})$ for $90-120 \mathrm{~min}$ to saturate the cortical surface within the cranial window. Surplus dye was washed out over 30-40 min with aCSF (Fig. 4). Subsequently, data acquisition was initiated and a 10-min baseline was acquired in both models.

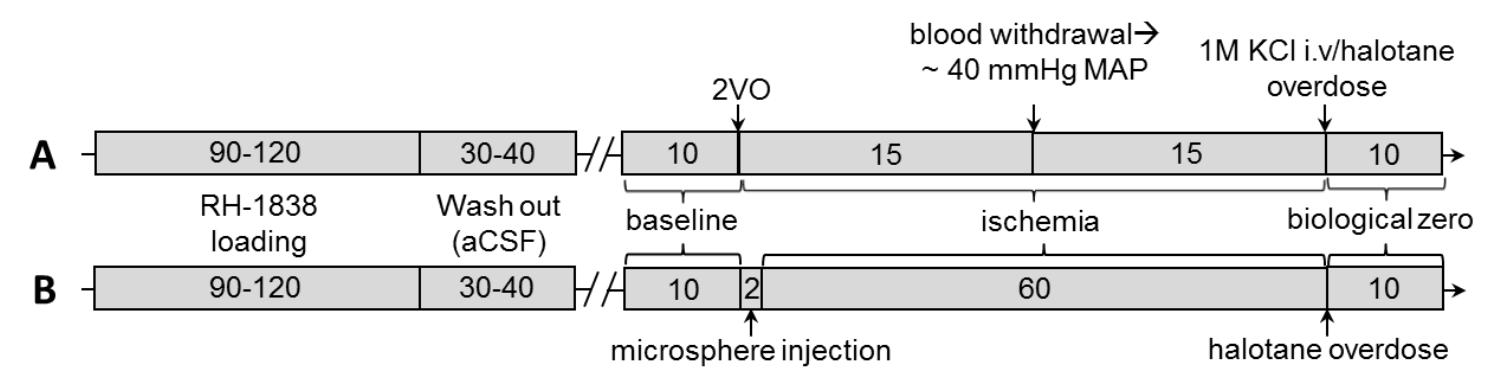

Figure 4: Timelines of experimental protocols for the two cerebral ischemia models (incomplete global forebrain ischemia model in (A) and microembolic stroke model in (B)). Duration of distinct periods is given in minutes.

\subsubsection{Incomplete global forebrain ischemia model}

2VO was produced by pulling and fixing the surgical thread looped around the common carotid arteries at both sides. The successful induction of $2 \mathrm{VO}$ was confirmed by an increase in MAP due to baroreceptor reflex. Cerebral ischemia was enhanced $10 \mathrm{~min}$ later by hypovolemic hypotension, produced by the gradual withdrawal of blood via either the femoral venous or arterial catheters, until MAP decreased to $40 \mathrm{mmHg}$, then stabilized between $35-45 \mathrm{mmHg}$ by mobilizing reserve blood. Each experiment was terminated 20 min later by either induction of sudden cardiac arrest (i.v. injection of 0.5 $\mathrm{ml}$ of $1 \mathrm{M} \mathrm{KCl}$ ), or halothane overdose. Recording was pursued 10 min after the induction of cardiac arrest (Fig. 4). 


\subsubsection{Microembolic stroke model}

Multifocal ischemia was induced by injecting 45-53 um diameter microspheres (UVPMS-BY2 45-53um, Cospheric, Santa Barbara, CA) suspended in 0,02\% tween 20 solution (2000 particles/ $0.6 \mathrm{ml}$ vehicle) via the ICA cannule, at a rate of $300 \mathrm{ul} / \mathrm{min}$. Optical signals were continuously captured for $60 \mathrm{~min}$ after ischemia induction. Each experiment was terminated by halothane overdose, and $10 \mathrm{~min}$ biological zero was recorded after cardiac arrest (Fig. 4).

\subsection{Multi-modal imaging}

A multi-modal imaging system, designed previously in our laboratory [45, 49], was applied to reveal SD-related changes in transmembrane potential and the coupled hemodynamic response in the cortical area under study (Fig. 5).

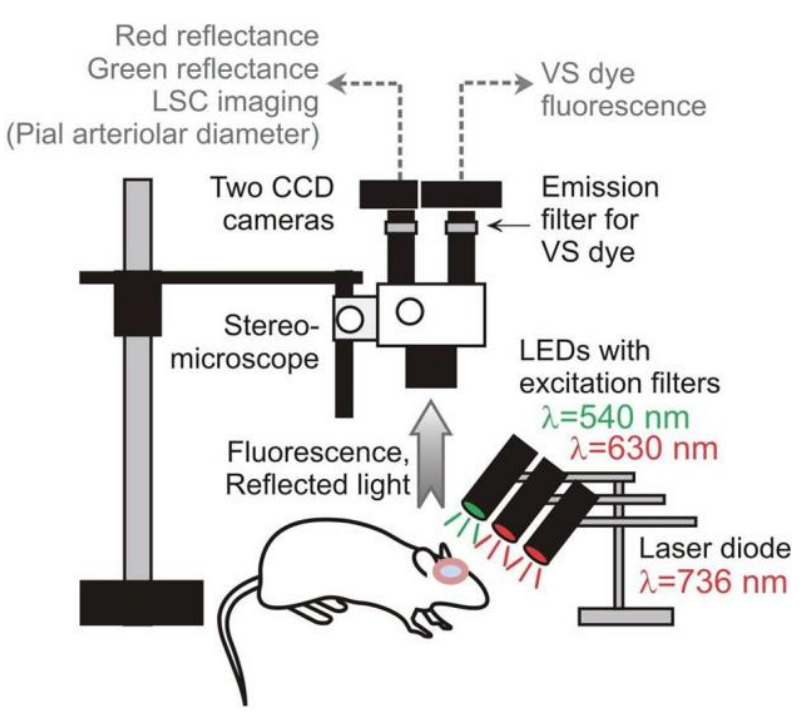

Figure 5: Schematic drawing of the setup used for multiple illumination and image capture in these experiments. The cranial window was illuminated in a stroboscopic mode by three distinct light sources: (i) A red LED (ii) a green high-power LED operated with a band-pass filter centered on the isosbestic point of hemoglobin; and (iii) a laser diode (Sanyo DL7140-201S; $70 \mathrm{~mW} ; 736 \mathrm{~nm}$ emission wavelength) driven by a power supply (ITC502, Thorlabs Ltd., Cambridge, UK) set to deliver a $100-\mathrm{mA}$ current. The alternating fluorescent and reflected light passed through a stereomicroscope on which 2 CCD cameras were mounted. Camera 1, equipped with a bandpass filter (670-740 nm), acquired only VS dye fluorescence sequences, while Camera 2 recorded LSC images and IOS under red and green illumination (final magnification of images: $\times 3.15)$.

At carefully selected illumination and strict timing of triggering-image capture (Fig. 6), optical signals (i.e. variations in light emittance and reflectance) related to changes in membrane potential and hemodynamic variables were visualized with a good spatiotemporal resolution.

\subsubsection{Imaging of cellular membrane potential changes}

To detect membrane potential changes, a VS dye (RH-1838, Optical Imaging Ltd., Rehovot, Israel ) that binds to cell membranes, and whose fluorescence increases with reduced cellular membrane potential [46] was used. To capture VS fluorescence images, 
the cortical surface was illuminated with a flashing red high-power light emitting diode (LED; 625 nm peak wavelength; SLS-0307_A, Mightex Systems, Pleasanton, CA) fitted with a 620-640 nm excitation filter (3RD620-640, Omega Optical Inc., Brattleboro, VT). Images were captured with a monochrome CCD camera i.e. Camera 1 (100 ms exposure each second; maximum resolution of $1024 \times 1024$ pixel; Pantera 1 M30, DALSA, Gröbenzell, Germany, equipped with a 3RD 670-740 nm emission bandpass filter (Omega Optical Inc) attached to a stereomicroscope (Figs. 5, 6).

\subsubsection{Imaging of cerebral blood flow changes}

Cerebral blood flow ( $\mathrm{CBF}$ ) was monitored at the exposed cortex with laser speckle contrast (LSC) imaging. The cortex was intermittently illuminated with a laser diode (Sanyo DL7140-201S; $70 \mathrm{~mW} 7736 \mathrm{~nm}$ emission wavelength). The raw laser speckle images were captured by a second CCD camera, identical to that used for VS dye imaging, and attached to the same stereomicroscope by using a 1:1 binocular/video-tube beam splitter (Figs. 5, 6). CBF maps were calculated with Image-Pro-Plus software (Media Cybernetics UK, Marlow, UK) from the obtained raw speckle images [47].
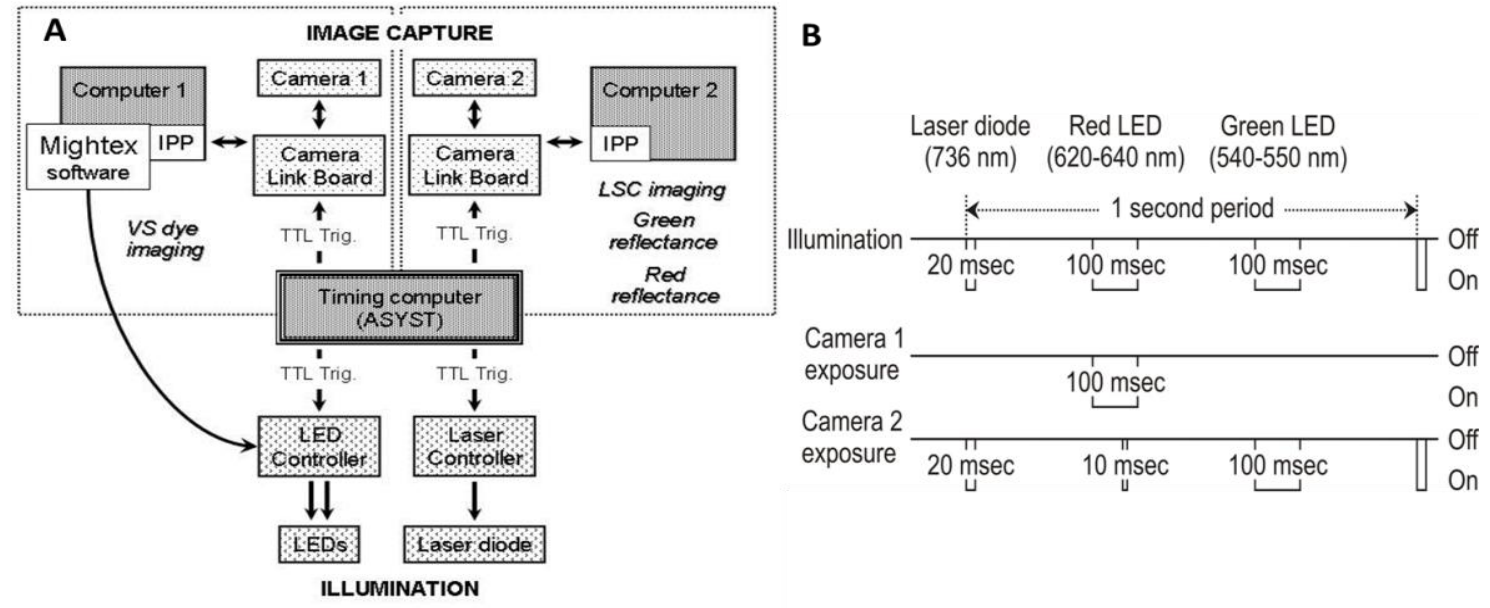

Figure 6: Diagram of the setup (A) and triggering (B) used for multiple illumination and image capture in these experiments. Synchronization of the illumination/image capture was achieved by the 'Timing' computer (A).

\subsubsection{Imaging changes in cerebral blood volume and hemoglobin saturation}

To reveal information about cerebral blood volume (CBV) and hemoglobin $(\mathrm{Hb})$ 
deoxygenation in the observed area, intrinsic optical signals (IOS) were captured with Camera 2. CBV in the cortex was assessed using IOS evoked at 540 to $550 \mathrm{~nm}$ green light illumination (100 msec illumination and $100 \mathrm{msec}$ exposure): decreasing green IOS intensity (i.e., decreasing intensity of reflected light) was expected to correlate with increasing CBV. In addition, changes in extracellular volume associated with SD were expected to contribute to variations in green IOS signal intensity (Fig. 7) [45, 55]. Hemoglobin deoxygenation was recorded with the help of IOS under 620 to $640 \mathrm{~nm}$ red light illumination (100 msec illumination, $10 \mathrm{msec}$ exposure): decreasing red IOS intensity represented decreasing $\mathrm{Hb}$ saturation; in case $\mathrm{Hb}$ was totally desaturated, red IOS intensity changes followed that of CBV.

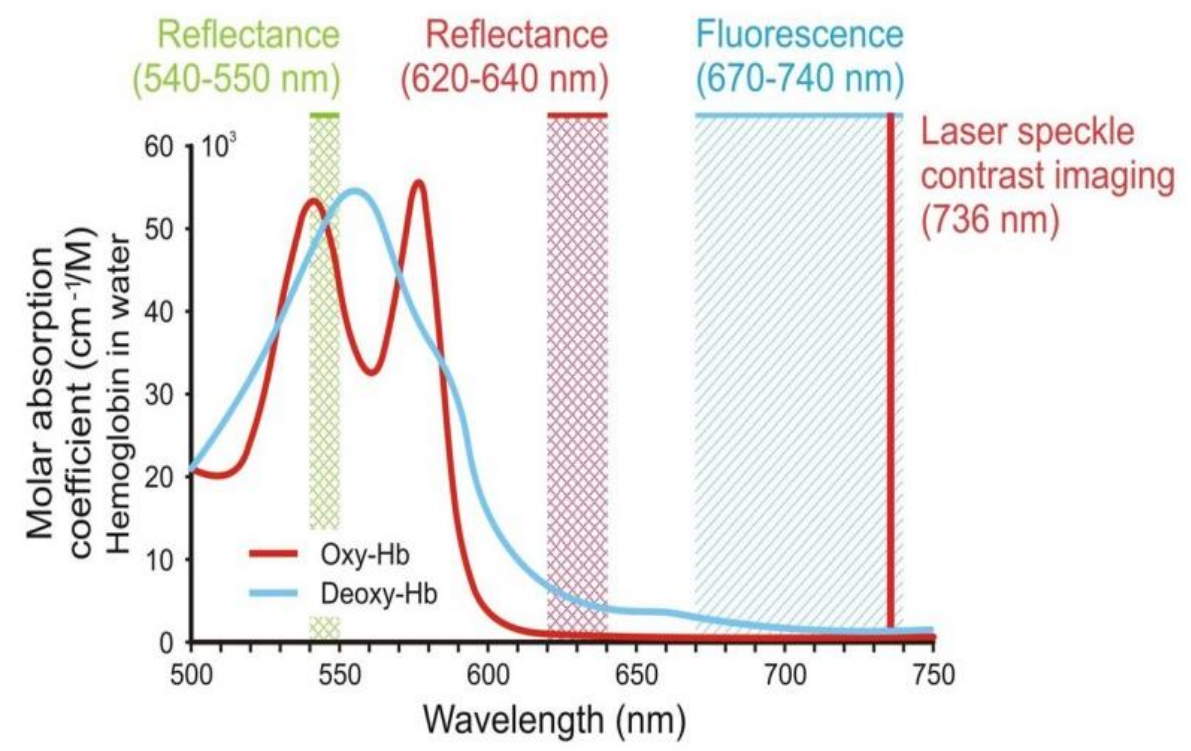

Figure 7: Specifications of the different illuminations used in the experiments (green and red LED; laser diode), of the VS dye fluorescence emission filter, and their relation to the 'absorbance' spectra of oxy$\mathrm{Hb}$ and deoxy-Hb. Values for deoxy-Hb and oxy-Hb molar extinction coefficients were taken from, http://omlc.ogi.edu/spectra/haemoglobin (i.e. 'compiled by Scott Prahl using data from: W. B. Gratzer, Med. Res. Council Labs, Holly Hill, London; and N. Kollias, Wellman Laboratories, Harvard Medical School, Boston'). The plot shows that both oxy- and deoxy-Hb absorb 540-550 nm green light equally (isosbestic point), therefore green IOS is expected to primarily indicate changes in local CBV (i.e., decreasing intensity of reflected light indicates CBV increase).

\subsubsection{Pial arteriolar diameter}

Pial arteriolar diameter was measured on selected arteriolar segments (average baseline diameter: $100 \mu \mathrm{m}$ ) in the incomplete global forebrain ischemia model. Measurements were carried out on green ISO images, at a resolution of 1024 x1024 pixels. After background normalization, a dedicated software (ImagePro Plus macro) calculated arteriolar diameter in each image of a sequence, based on automatic edge detection between dark and light areas (dark area-vessel, light area-background tissue). 


\subsection{Protocol for image analysis}

Local changes in VS dye fluorescence, CBF and IOS at red and green illumination with time were determined by positioning selected areas of interest (AOI, $3 \times 3$ pixel size in the incomplete global forebrain ischemia model, $6 \times 6$ in the microembolic stroke model) on the image sequences in such a way that visible blood vessels were avoided. The position of AOI was random for general data assessment, and carefully selected when the focus of an SD or distinct SD evolution was analyzed (microembolic stroke model). The average gray level within an AOI was measured in individual frames constituting each image sequence, and plotted against the corresponding time of data acquisition. Once assembled, these data provided the corresponding kinetic of changes in fluorescence intensity, CBF and IOS throughout the recording period.

\subsection{Histology in microsphere model}

To confirm ischemic lesion evolution, 7 male Sprague-Dawley rats (360 to $430 \mathrm{~g}$ ) were anesthetized with $1.5 \%$ to $2.0 \%$ isoflurane in $\mathrm{N}_{2} \mathrm{O}: \mathrm{O}_{2}(2: 1) 24$ hours after microsphere embolism, and decapitated. Brains prepared for slicing were rinsed in icecold $0.1 \mathrm{M}$ phosphate-buffered saline, and were sectioned in the coronal plane with microtome blades in a rodent brain matrix. Brain slices $(2 \mathrm{~mm}$ thick) were incubated in 2,3,5-triphenyltetrazolium chloride (TTC) in $0.1 \mathrm{M}$ phosphate-buffered saline for $20 \mathrm{~min}$ at room temperature, than stored in $4 \%$ paraformaldehyde. Because TTC staining may not be sensitive enough to detect microinfarcts, the TTC-stained sections were latter embedded in paraffin, sectioned to $5 \mu \mathrm{m}$-thick slices, stained with hematoxilyyn/eosine (HE), and examined with optical microscopy. Sections were digitally recorded with a microscope slide scanner (Zeiss Mirax Midi Slide Scanner, Carl Zeiss Microimaging $\mathrm{GmbH}$, Jena, Germany). 


\section{Results}

\subsection{Changes in the acquired variables with ischemia induction}

\subsubsection{Global forebrain ischemia}

After $2 \mathrm{VO}$ onset, baseline MAP $(88 \pm 4 \mathrm{mmHg}$ ) suddenly dropped to $70 \pm 4 \mathrm{mmHg}$ and then immediately increased to $107 \pm 6 \mathrm{mmHg}$, which is considered to be the sign of intact baroreceptor reflex. MAP then persisted at a higher level $(91 \pm 4 \mathrm{mmHg})$ until the initiation of hypovolemic hypotension. With gradual blood withdrawal, MAP reduced progressively, then persisted at $40 \pm 4 \mathrm{mmHg}$ until the termination of the experiment (Fig. 8)

CBF varied in a similar fashion and with a minimal latency with respect to MAP. Following a brief perfusion drop immediately after $2 \mathrm{VO}$ onset $(65 \pm 5 \%)$, CBF increased parallel with MAP and persisted at around $78 \%$, then reduced with blood withdrawal $(39 \pm 5 \%)$ and settled on near this value (Fig. 8).

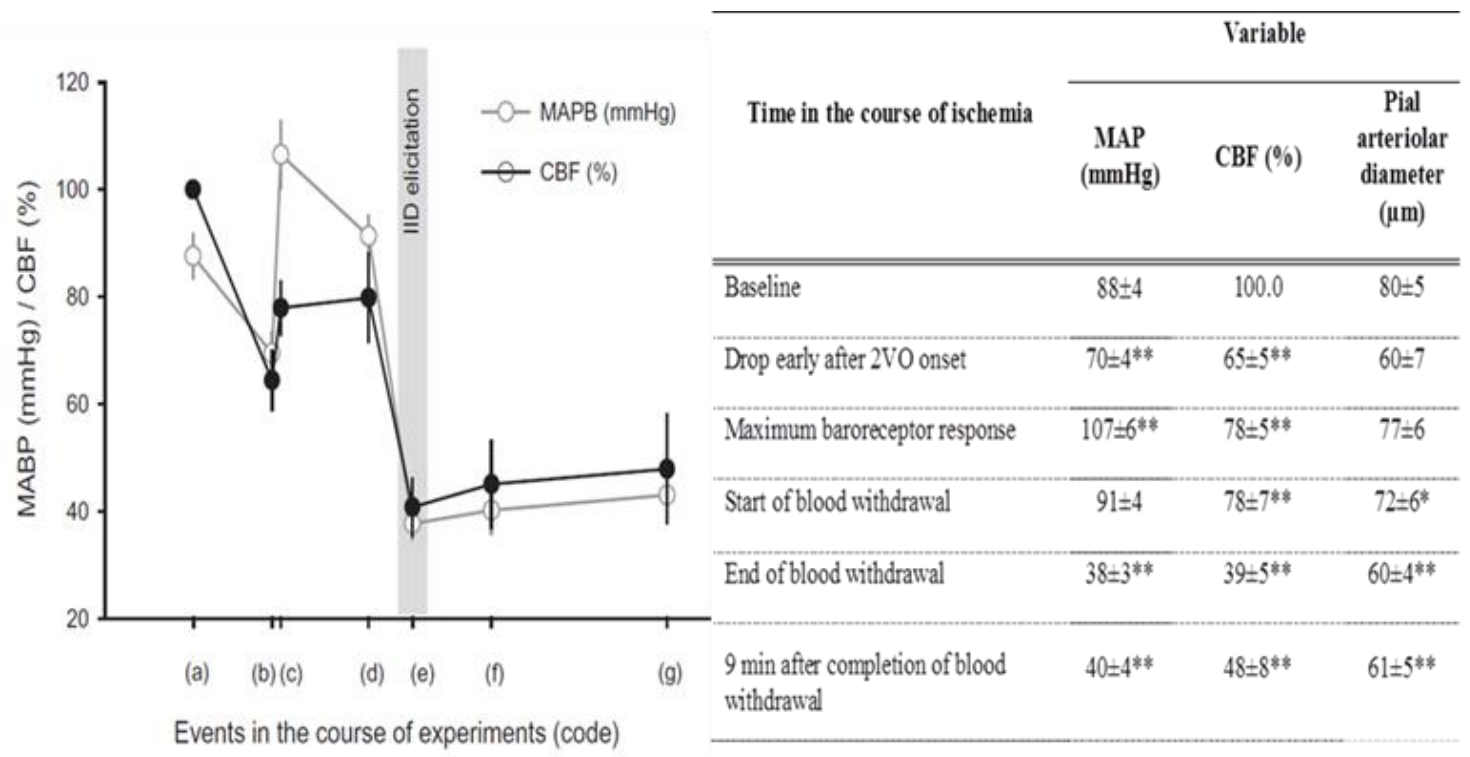

Figure 8: Changes in systemic caridovascular and cerebrovascular conditions over the course of the experimental protocol. Events in the course of experiments: (a) baseline, (b) early after 2VO onset, (c) max. baroreceptor response, (d) start of blood withdrawal (e) end of blood withdrawal (f) 9 min after the completion of blood withdrawal $(\mathrm{g})$ immediately before cardiac arrest. Grey column represents the period during which ischemia induced depolarisation occurred (ie. SD). Table shows changes in mean arterial pressure (MAP), cerebral blood flow $(\mathrm{CBF})$, and the diameter of selected pial arterioles. Data are expressed as mean \pm SEM. Significant changes over time were assessed with analysis of variance (ANOVA) with repeated measurements. ( $* * \mathrm{P}<0.01$ and $* \mathrm{P}<0.05$ given as levels of confidence) Abbreviations: 2VO: bilateral common carotid artery occlusion (2-vessel occlusion), SD: peri-infarct depolarization. 
Transient drop of pial arteriolar diameter of selected vessels was detected due to $2 \mathrm{VO}$ onset; from a baseline of $80 \pm 5 \mu \mathrm{m}$, arteriolar diameter reduced to $60 \pm 7 \mu \mathrm{m}$, and subsequently dilated to $77 \pm 6 \mu \mathrm{m}$. At the end of blood withdrawal, arteriolar caliber remained at around $60 \mu \mathrm{m}$ (Fig. 8)

A sudden negative shift in VS dye signal intensity developed with $2 \mathrm{VO}$ onset in all experiments $(-4.6 \pm 0.8 \mathrm{gl} \%, \mathrm{n}=13)$. In 7 cases, slight elevation in VS dye signal developed immediately after the negative shift and an additional drop of VS dye fluorescence intensity was detected at the initiation of hypovolemic hypotension ($3,90 \pm 0.6 \mathrm{gl} \%)$.

Green IOS suddenly increased $(7.50 \pm 0.7 \mathrm{gl} \%)$ immediately after $2 \mathrm{VO}$ onset, in accordance with the marked reduction of CBF. A similar positive shift in green IOS occurred in the course of the development of hypovolemic hypotension $(6.87 \pm 1.4 \mathrm{gl} \%)$.

Red IOS dropped with $2 \mathrm{VO}$ onset $(-5.86 \pm 0.8 \mathrm{gl} \%)$, and also with the initiation of hypovolemic hypotension $(-4,58 \pm 0.9 \mathrm{gl} \%)$.

\subsubsection{Multifocal ischemia}

Embolisation (i.e. ischemia induction) was considered successful, if the presence of microsphere could be confirmed in pial arterioles revealed by the cranial window. Successful ischemia induction was also confirmed by following microsphere capture in videos acquired at green light illumination (Fig. 9 A-B) and by monitoring CBF. Immediately after microsphere infusion, CBF dropped distal to the occlusion in selected pial arterioles. This is demonstrated by a representative case in Figure 9 C1-C3.

$\mathrm{CBF}$ in the cortical parenchyma revealed by the cranial window showed a heterogenous reduction (Fig. 10). As Figure 10 demonstrates, CBF dropped below 50\% in $8 \%$ of the cortical area; was maintained between $50-90 \%$ about $\sim 61 \%$ of the cortex, and stayed above $90 \%$ in around $30 \%$ of the visualized area.

After the termination of the experimental protocol, brains were removed, and fluorescent images covering the entire dorsal brain surface were obtained at UV illumination. These images revealed that microspheres blocked pial arterioles on the ipsilateral hemisphere randomly, and at multiple sites of the observed area (Fig. 11). This was expected to cause multifocal ischemia. 

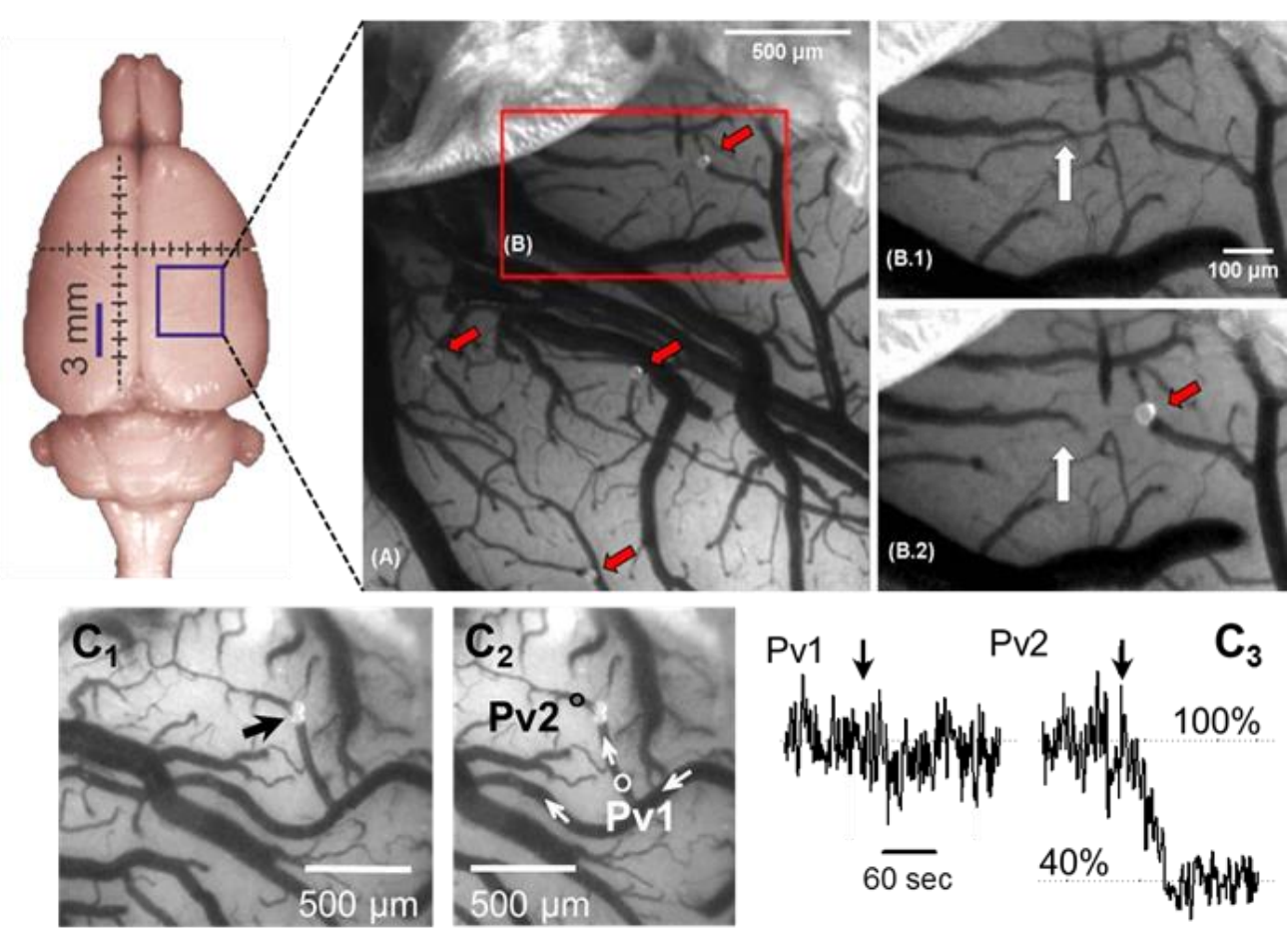

Figure 9: Representative images and graphs illustrate the reduction in blood flow in the pial arterioles after microsphere capture. Pictures were taken at green $(\lambda=540-550 \mathrm{~nm})$ illumination. (A) Anatomy of the parietal cortex revealed by the cranial window. Red arrows indicate microspheres in pial arterioles. Picture (B.1 was taken before microsphere administration-blood flow is undisturbed in a pial arteriole (white arrow). Picture (B.2) was taken $2.5 \mathrm{~min}$ after microsphere administration. Microsphere (red arrow, $d=45$ to $56 \mu \mathrm{m}$ ) blocks blood flow in the same vessel (white arrow). C1-2: a selected pial arteriole with captured microspheres. Blood flow detected in selected area of interest i.e. AOI (small circles), placed on the vessels proximal (Pv1) and distal (Pv2) to the obstruction. The direction of blood flow is shown by white arrows. C3, Traces were obtained by laser speckle contrast analysis (LSC), and represents the kinetics of blood flow change in the selected AOI. The time of microsphere capture is marked with vertical arrow. Proximal to the obstruction (Pv1 AOI) blood flow remained constant. Distal to the blockade (Pv2 AOI) blood flow dropped (100\% to 40\%) and persisted at a low level.

In order to confirm that microembolisation did cause brain infarction, TTC stained brain slices prepared $24 \mathrm{~h}$ after ischemia induction were investigated. Figure 12 A1 shows a focal lesion in the cortex (pointed at by arrow, $n=2$ ) while Figure 12 A2 demonstrates more diffuse cortical infarctions $(n=3)$ in the ipsilateral cortex. Ischemiarelated lesions were not detected in 2 rats. In these two cases, probably microsphere infusion failed to reach the brain, instead, microspheres were possibly drained into the pterygopalatinal artery. 


\subsection{Characterization of spreading depolarizations and associated hemodynamic responses}

\subsubsection{Global forebrain ischemia}

Experiments fell into 3 categories based on whether depolarization was elicited successfully, and if so, during which phase of the experimental procedures SD appeared; (i) depolarization did not evolve during the period of recording $(n=4)$, (ii) depolarization emerged at a time during blood withdrawal carried out to enhance forebrain ischemia by hypovolemic hypotension $(n=3)$ and (iii) depolarization occurred after forebrain ischemia had been completed by hypovolemic hypotension $(n=4)$. No SD emerged under $2 \mathrm{VO}$ alone, or during the $10 \mathrm{~min}$ between $2 \mathrm{VO}$ and blood withdrawal. Average time to $\mathrm{SD}$ appearance with respect to the initiation of hypovolemic hypotension was $4.6 \pm 0.8 \mathrm{~min}$.

SDs entered and propagated as a single wave front across the cranial window without attenuation, originating from a cortical area that fell out of the field of view (Fig. 13). A single SD was detected in each experiment, which invaded the cranial window predominantly from the fronto-lateral corner of the observed area and progressed to caudo-medial direction ( $\mathrm{n}=8$ of 9 experiment), in one case, propagation was retrograde (i.e caudo-lateral fronto-medial). Average propagation rate was $2.8 \pm 0.2$ $\mathrm{mm} / \mathrm{min}$.

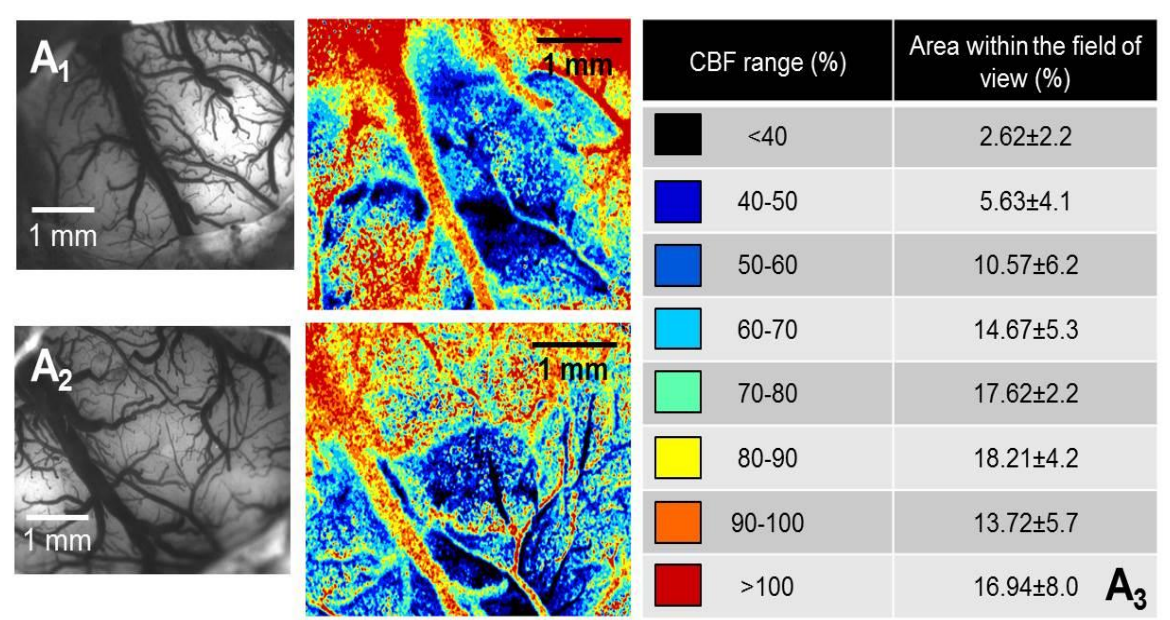

Figure 10: Whole field analysis of CBF after ischemia induction. Panel A1 and A2: vascular and macroscopic anatomy of cortical area within the field of view revealed at green illumination $(\lambda=540-550$ $\mathrm{nm})$. Colored flow maps next to photomicrographs in A1 and A2: cortical blood flow relying on laser speckle contrast (LSC) analysis,; false-colored CBF maps reveal the heterogeneity of ischemia-related CBF reduction. Note the uneven color distribution-. Panel A3 shows the average ratio of surface area of defined perfusion ranges (mean $\pm \mathrm{SD} n=7$ ) 
Based on the kinetics of the wave front and recovery of transmembrane potential, three SD types were established (Fig. 14):

Persistent/terminal SDs $(n=4)$ : sudden, profound depolarization, without recovery of transmembrane potential during the observation period, similar to anoxic depolarization (AD), typical of the ischemic core, or depolarization seen after cardiac arrest in the rat [49]. CBF decrease was sustained in these cases (by $11.7 \pm 5.1 \%, \mathrm{n}=4$ ).

- Intermediate/prolonged SD waves $(\mathrm{n}=3)$ : sudden depolarization, and delayed recovery of transmembrane potential (depolarization was longer than 5 min) [32], coupled with transient CBF reduction (by $17.4 \pm 5.2, \mathrm{n}=3$ )

- Transient SD waves $(n=2)$ : transient depolarization, with immediate recovery of transmembrane potential, but longer duration $(37 \pm 13 \mathrm{~s})$ compared to SDs in non-ischemic cortex (14-16 s) [25]. This SD type was typically associated with no flow response i.e. CBF stabilized at the pre-event level.

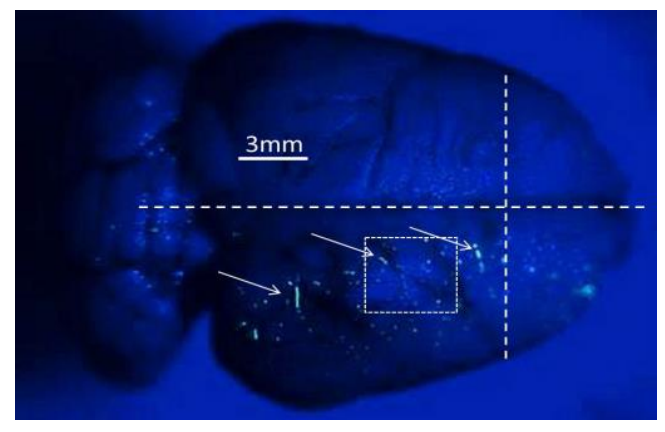

Figure 11: Microsphere infused rat brain under $365 \mathrm{~nm}$ UV light (i.e. the excitation wavelength of fluorescent microspheres; emission: yellow/green light). Horizontal dash line represents the longitudinal fissure, vertical dashed line depicts the level of Bregma. Rectangle on the right parietal hemisphere represents the position of the cranial window. Green spots pointed at by arrows are microspheres, captured in arterioles. Note the uneven dispersion of microspheres on the cortical surface. Note, that microspheres occur only in the ipsilateral hemisphere.

IOS changes occurred simultaneously with SD waves. The intensity of both red and green IOS was suddenly increased with transient and intermediate SD occurrence (Fig. 14 BC). Recovery of red and green IOS was incomplete but simultaneous with repolarization for these SD types (Fig. 14 B-C). In contrast, IOS intensity remained elevated (i.e. no partial recovery) with persistent SDs (Fig. 14 A).

\subsubsection{Multifocal ischemia}

In total, 31 SDs were recorded in 7 experiments. All the observed SDs appeared to be related to ischemia, since they occurred after the induction of multifocal ischemia. The number of SDs during the period of observation (i.e., 1 hour) ranged from 2 to 12 events per experiment. The foci of five SDs were seen within the cortical area revealed by the cranial window; all the other SDs invaded the area from a distant origin. 


\subsubsection{Focal events and associated hemodynamic changes}

Foci of five SDs were identified within the cranial window in four different rats: in one rat, two SD foci were seen (first and fourth SDs). All the other depolarization waves originated from further cortical areas. The VS dye signal extracted at the proper SD focus indicated three different SD types similar to those observed in the global forebrain ischemia model above short, transient SD $(n=3)$, intermediate SD $(n=1)$ and persistent SD $(n=1)$. Intermediate and persistent waves converted to short transient waves and propagated in radial manner to further cortical regions. Propagation rate reduced from $6.5 \pm 4 \mathrm{~mm}$ (focus) to $3.9 \pm 1.6 \mathrm{~mm} / \mathrm{min}$ ( $\sim 1 \mathrm{~mm}$ to focus) during conversation. Figure 15

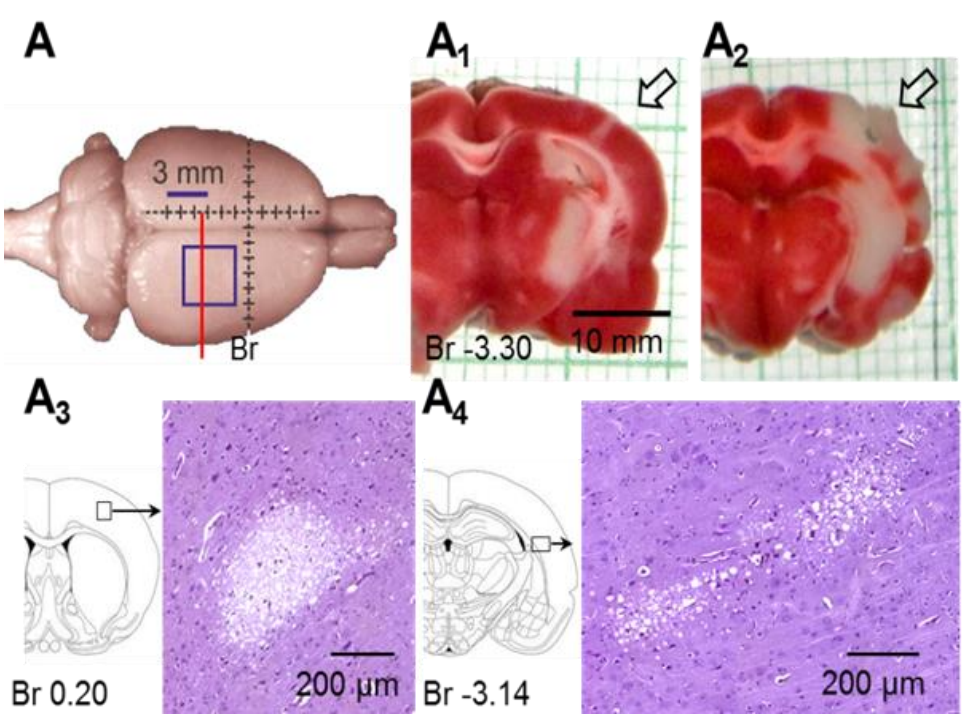

Figure 12: Histological assessment of brain infarction $24 \mathrm{~h}$ after microsphere infusion. A: blue rectangle on right parietal hemisphere of the rat brain shows the position of the cranial window. Red line represents the plane of 2,3,5triphenyltetrazolium chloride (TTC)-stained coronal brain sections. A1-A2: brain slices after TTC staining, arrow is pointing at focal (A1) and extensive (A2) cortical ischemic lesions. A3-A4:

Hematoxylin/eosin staining reveals cortical microinfarcts.

The CBF signal taken at carefully selected AOIs positioned at the SD focus and at increasing distances from the focus indicated a gradual conversion of the SD-associated $\mathrm{CBF}$ response as SD propagated. Local CBF measured shortly before SD evolution was the lowest at the focus of the latter SD and increased with the distance; in a particular case, $\mathrm{CBF}$ was as high as $86.9 \pm 18.7 \% 1 \mathrm{~mm}$ away from the SD focus, with respect to $56.9 \pm 9 \%$ at the proper focus (Fig. 16 C). At the SD focus, classic hyperemic response failed to evolve; either minor hyperemia $(n=2)$, or no flow response $(n=3)$ were detected with depolarization events, irrespective of the type of the SD. The magnitude of SDrelated hyperemia increased with the distance from the SD focus (Fig. 16 D). Cerebral blood flow returned to the pre-SD level after the passage of the hyperemic response 

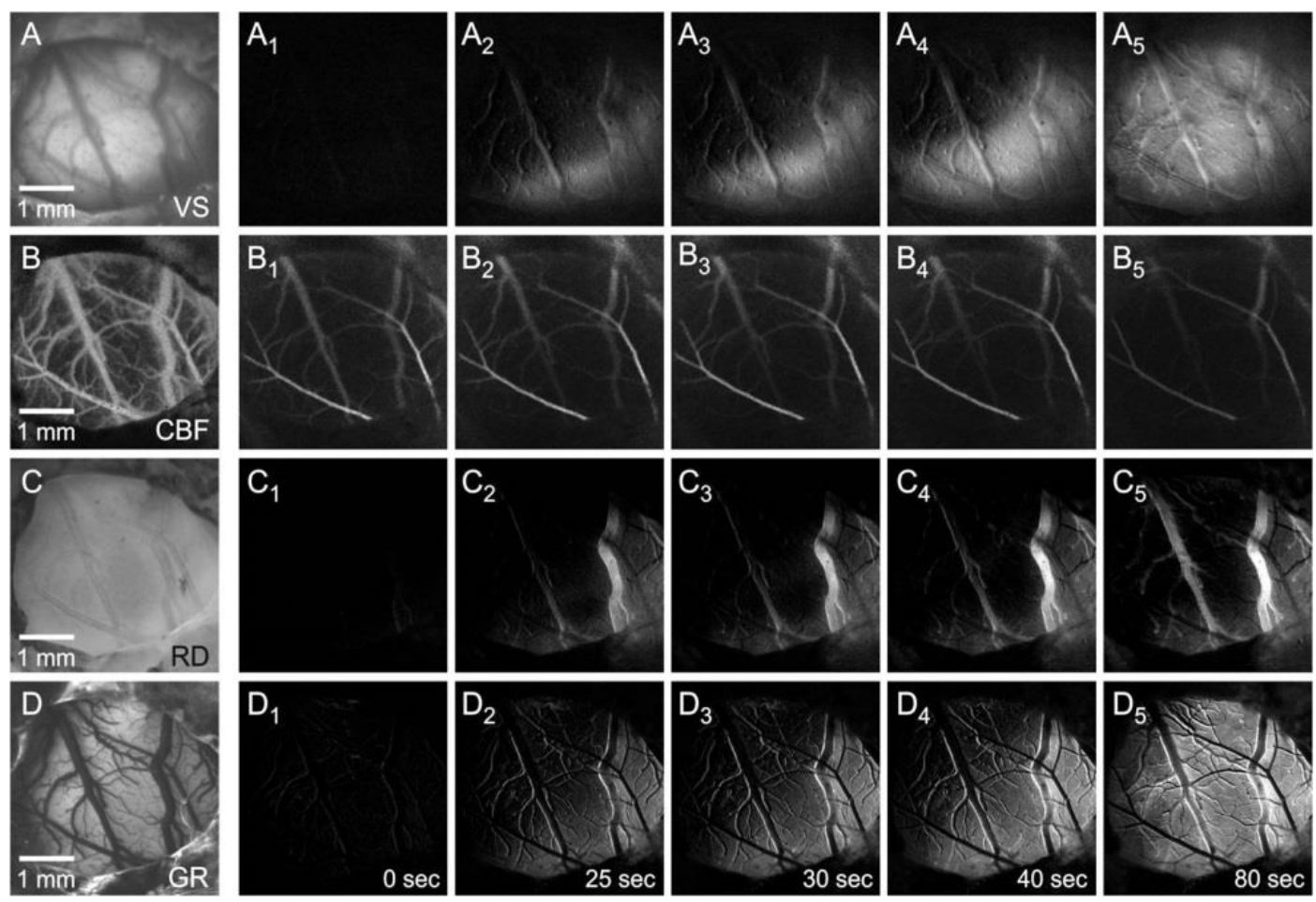

Figure 13: Representative image sequences, and kinetics of changes in the four variables under study, recorded during the progression of SD. In this particular experiment, depolarization was intermediate, membrane potential was restored after $13 \mathrm{~min}$ and, the SD was associated with hypoemic flow response. Pictures A, B, C, \& D show the cortical region under study at the various illuminations, with images taken shortly after tissue loading with the VS dye. Panels A1-A5 represents voltage sensitive (VS) dye fluorescence intensity i.e. membrane potential change. In this case, the SD wave invaded the cranial window from a fronto-lateral direction (A2) and propagated to caudo-medial direction (A3-A5). Panels B1-B5 display laser speckle contrast (LSC) images of cerebral blood flow (CBF) - the darker the area the lower the blood flow. Quantitative analysis of the SD associated CBF variation showed prolonged CBF reduction. Panels C1-C5 demonstrate alterations of the red intrinsic optical signal (RD, hemoglobin deoxygenation). The green intrinsic optical signal (GR, cerebral blood volume; D1-D5) suggested persistent reduction of cerebral blood volume.

\subsubsection{Features of propagating spreading depolarizations and the associated cerebral blood flow response}

The majority of the detected SDs displayed short, transient changes of membrane potential (i.e. VS dye signal), and invaded the area from a distant origin. Most of the SD waves originated from the frontal-frontolateral site $(n=19), 5$ SD events originated from lateral edge of the window, and 2 SD waves travelled from the caudal direction. Propagation rate varied between 1.8 to $6.2 \mathrm{~mm} / \mathrm{min}(3.02 \pm 1.08 \mathrm{~mm} / \mathrm{min}$ as average). The site of SD entry to the cranial window was not conserved within individual experiments; in addition, propagating SDs were interspersed with SDs emerging in the cranial window, implying multifocal SD origin in the ischemia model used.

Several SDs gradually extinguished over their course of propagation through the 
observed area. In these cases, CBF was relatively homogeneous within the field of view at the time of SD elicitation $(75.3 \% \pm 12.5 \%$ with respect to baseline $100 \%)$ Typically, hyperemic CBF response was coupled with SD waves (peak of hyperemia 129.8 $\pm 18.6 \%$ ). Peak hyperemia was the highest $(130 \%)$ at the site where the wave front entered the visualized area, and became gradually less pronounced as the wave advanced over the cortex (Fig. 17). At the most distant sampling site with respect to SD entry to the cranial window, the VS dye signature of SD did not indicate any shift in membrane potential, and the associated CBF signal showed no variation, either. The changes in green and red IOS evolved parallel with the shifts in VS dye fluorescence and CBF: the SD-related decrease in green and red IOS (increase in $\mathrm{CBV}$ and $\mathrm{Hb}$ desaturation, respectively) became gradually smaller over the course of SD propagation.
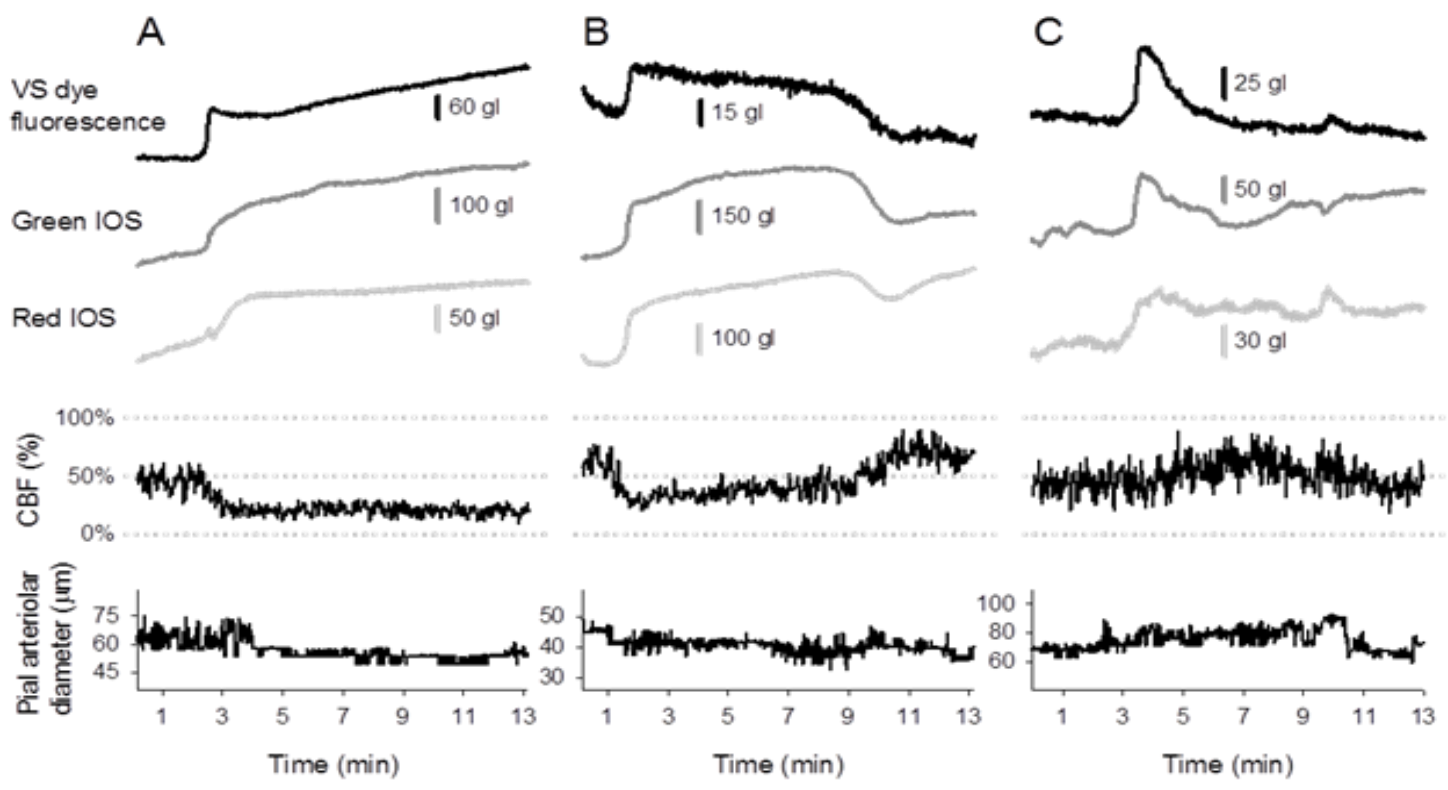

Figure 14: Different types of depolarization waves and associated hemodynamic responses. Simultaneous kinetic of changes in voltage sensitive (VS) dye fluorescence (i.e. membrane potential change), red intrinsic optical signal (IOS), green IOS, cerebral blood flow (CBF), and diameter of a selected pial arteriole during SD recorded in three representative experiments. Panel A: irreversible, negative shift in membrane potential (i.e. persistent SD), coupled with a marked reduction in CBF, and a decrease of arteriolar caliber. Panel B: Prolonged SD with hypovolemic flow response. CBF recovered to pre-event level parallel with repolarization. Panel C: transient, negative shift in membrane potential followed by the recovery of resting membrane potential. The SD was accompanied by a slow, minor increase in local $\mathrm{CBF}$.

In 2 experiments, it was clearly seen in videos that several SDs curved around patches of the cortex as they propagated. Terminal depolarization was not detected in the regions avoided by SDs during image acquisition. In these particular experiments, CBF in the cortex estimated right before SD elicitation showed areas of higher and lower values 
ranging between $40 \%$ and $100 \%$ of baseline. In the representative example given in Figure 18, the incoming SD invaded the cranial window from a frontolateral direction, and curved around regions showing the lowest CBF ( $40 \%$ of baseline, deep blue in Fig. 18C).

Kinetics of SD related IOS changes are represented in Figure 19. Hyperemic CBF response to SD-s was typically accompanied by a decrease in green IOS as the sign of increase in CBV (increasing amount of hemoglobin absorbs more green light). The maximal reduction of green IOS coincided with the peak of the CBF response to SD. Red IOS changes related to SD appeared to be biphasic; the following phases were established: (i) an early increase in signal intensity ( $\mathrm{Hb}$ saturation) coincident with the initial phase of CBF elevation, (ii) a latter, sharp decrease in red IOS below baseline (Hb desaturation), the start of which preceding the peak of hyperemia. Based on the dominance of either of the two phases, the following types of red IOS signatures were defined: Type 1 dominant initial phase ( $\mathrm{n}=5)$ (Fig. 19 A); Type 2 equal magnitude of both phases (n=7) (Fig. 19 B); Type 3 dominant later phase (n=12) (Fig. 19 C). In the single case when hypoperfusion rather than hyperemia evolved with SD, red IOS showed a monophasic, marked reduction (i.e. Hb desaturation) (Type 4, Fig. 19 D).

\section{Discussion}

Ischemia-induced SD waves in hypoxic-ischemic brain injury contribute to the extension of tissue damage and predict worse neurological outcome [27, 28, 32]. Because of their well-defined pathological impact, SDs are extensively studied in patients of [17, 20,56] malignant hemispheric stroke, subarachnoid hemorrhage and traumatic brain injury. Still, monitoring SD evolution in the acute phase of ischemia in patients is not feasible due to the delay between injury onset, admission to hospital, and surgical alleviation of symptoms. Furthermore, SD monitoring can only be conducted in conditions requiring craniotomy. These limitations, and the complexity of the phenomenon still requires appropriate animal models and sophisticated methods to provide a comprehensive picture about the evolution of depolarization waves and neurovascular coupling in cerebral ischemia.

In our experiments, we characterized the evolution, propagation and kinetics of spontaneous depolarization waves and the coupled hemodynamic variables in the ischemic rat cerebral cortex. Further we identified ischemic perfusion patterns that favor 
SD occurrence. Finally, we recognized various types of neurovascular coupling with SD in global forebrain and multifocal cerebral ischemia. Going beyond classic one point electrophysiological measurements and perfusion monitoring (such as DC potential recording combined with Laser Doppler flowmetry, frequently used in animal stroke models), our multimodal imaging technique enabled the simultaneous detection and visualization of ischemia- and SD-related changes in transmembrane potential and the coupled hemodynamic response (i.e. local cerebral blood flow, blood volume, hemoglobin saturation) in a cortical area at high spatiotemporal resolution [49].

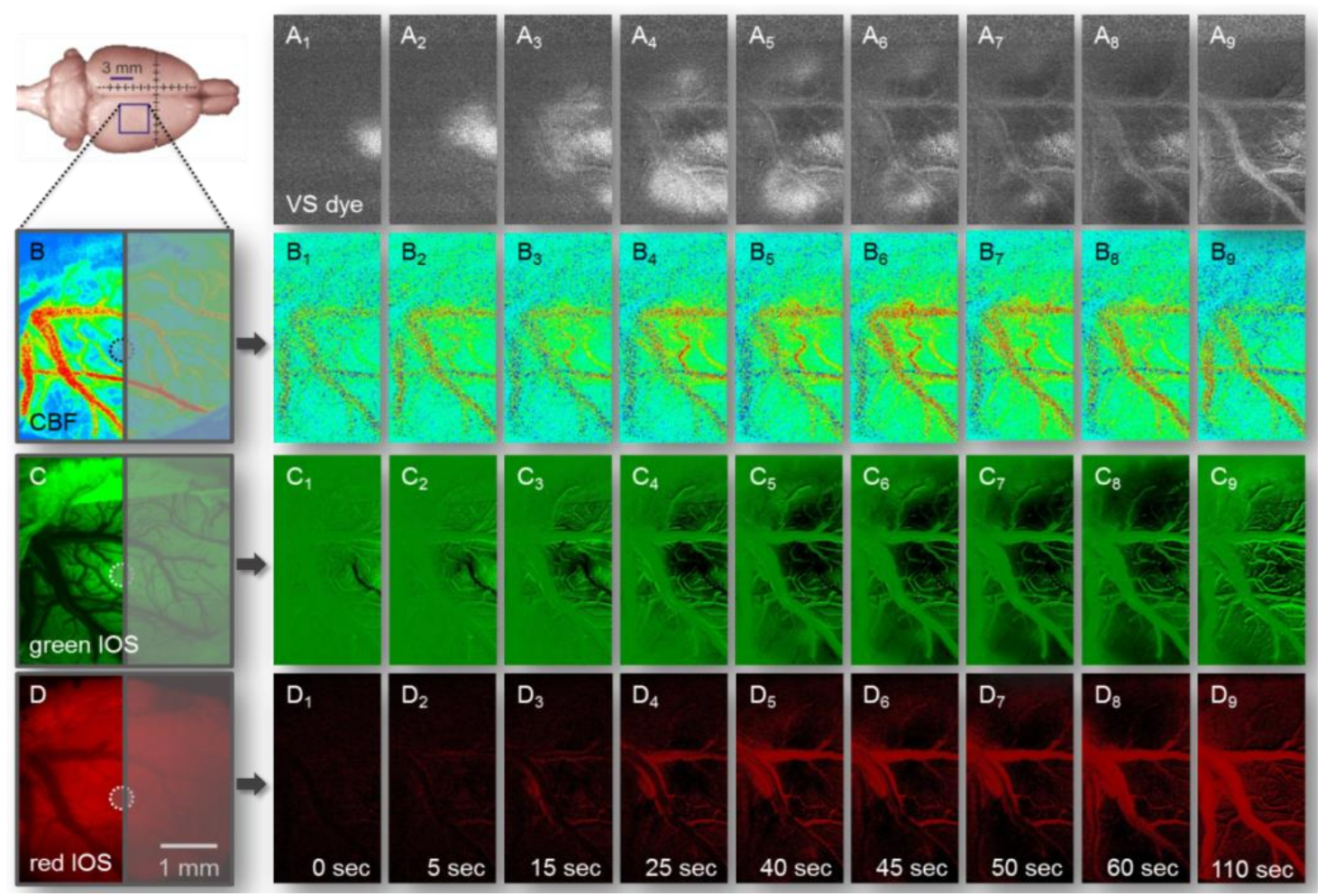

Figure15: Representative image sequences in the four variables under study, recorded during the initiation and propagation of a spreading depolarization (SD) event originating in the cranial window. The blue rectangle on the rat brain in the upper left hand corner depicts the position and size of the cranial window. Focus of an SD event is represented with dotted circle in B,C and D. Panels A1-A9 represent VS dye fluorescence intensity: SD focus appears as a light grey spot in A1, and SD propagates radially (i.e. to the left of subsequent images). Panels B1-B9: Laser specle contrast (LSC) images of cerebral blood flow (CBF). Panels C1-C9, SD-related variations in green intrinsic optical signal (IOS) represent alteration of cerebral blood volume $(\mathrm{CBV})$; D1-D9, SD-associated changes in red IOS indicating the level of hemoglobin saturation synchronously with SD. 


\subsection{The general impact of ischemia induction on mean arterial blood pressure and cerebral blood flow}

\subsubsection{Global forebrain ischemia model}

Our global forebrain ischemia model was used to show the effect of prolonged, stepwise ischemia development achieved by carotid occlusion in combination with persistent hypotension. This approach enabled the examination of the compensatory capacity of the circulation and exhaustion of these mechanisms: shortly after 2VO onset, transient elevation of MAP was detected, which was accepted as a sign of intact baroreceptor reflex [57]. The subsequent, progressive decrease of MAP with the gradual development of hypovolemic hypotension obviously reflected the reduction of systemic blood volume [58]. The sharp drop in CBF after 2VO onset confirmed the evident cessation of the anterior blood supply to the forebrain [59]. CBF persisted and stabilized under the pre-ischemic level coincident with the temporary elevation of MAP. The initiation of systemic hypotension caused a further decrease of CBF which followed MAP reduction. This, and the low level of CBF after completion of hypovolemic hypotension demonstrated, that the autoregulatory capacity of the cerebral circulation had been exhausted (i.e. vascular compensatory mechanisms against MAP fluctuation no longer maintained stable tissue perfusion).

Consistent with the assessed MAP and CBF variations, green and red IOS also varied: increasing green IOS intensity (i.e. decreasing light absorption by hemoglobin) with ischemia onset indicated mass hemoglobin amount/CBV reduction in the examined cortical tissue, while the simultaneous decrease in red IOS intensity (i.e. increased light absorption by elevated deoxy-Hb) with $2 \mathrm{VO}$ and the later induction of hypovolemic hypotension reflected hemoglobin desaturation [49, 60]. Mild VS dye intensity changes during ischemia induction were not related to membrane potential change: the fluorescence intensity of the dye was probably altered by the absorption of both its excitation wavelength and emittance by changing CBV and hemoglobin saturation [49].

\subsubsection{Multifocal cerebral ischemia model}

In the second study, the multifocal microsphere-induced stroke model modified to our specific purposes was applied to induce multiple ischemic foci in the brain. Magnetic resonance imaging studies already showed that CBF drops immediately after microsphere 
administration, but ischemic-injury progression is slower than in MCAO models, and the infarcts keep maturing for 24-48 hours after induction [53]. In our experiments, the perfusion deficit immediately after embolization and during the early phase (i.e. the first hour) of ischemia was similar to that previously described in the MRI study. Furthermore, as clear signs of ischemia-related tissue damage, extended cortical and subcortical infarctions were identified in TTC stained brain slices 24 hours after ischemia induction.
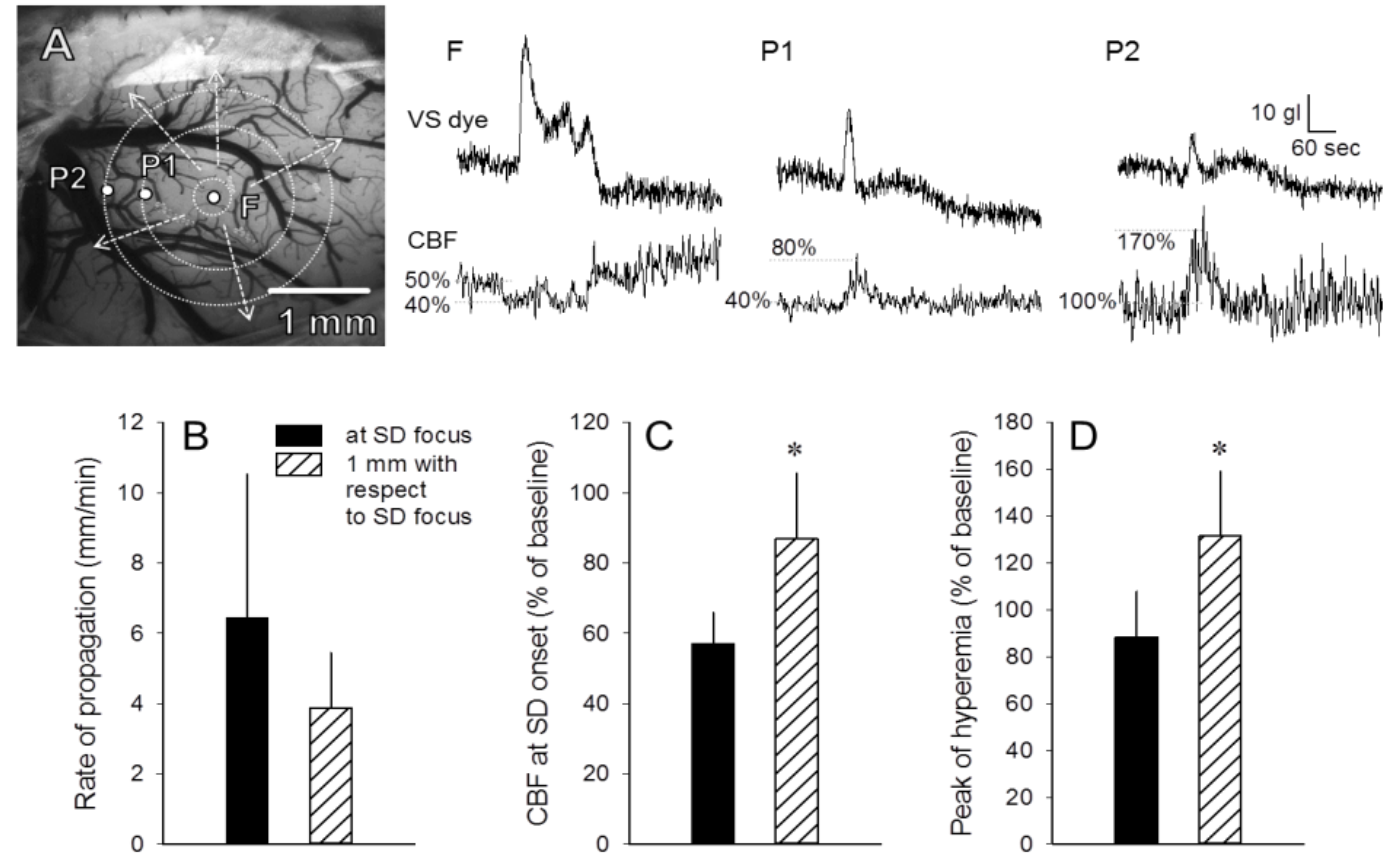

Figure 16: Kinetics of membrane potential change (VS dye) and associated cerebral blood flow (CBF) response in the focus of spreading depolarization (SD) and adjacent cortical areas. In Panel A, the image demonstrates the anatomy of the cortex under green illumination $(\lambda=540-550 \mathrm{~nm})$. SD focus $(F)$ and selected sampling sites near $(\mathrm{P} 1-1 \mathrm{~mm})$ and more distant $(\mathrm{P} 2-1.5 \mathrm{~mm})$ to the focus are shown. Arrows indicate the radial direction of propagation. The traces of VS dye fluorescence intensity and CBF illustrate the evolution of a representative spreading depolarization taken at the focus of SD (F), and at two selected points (P1 and P2) in the cortex. Sustained flow reduction to depolarization in the focus converted to transient hyperemia $1 \mathrm{~mm}$ further. While the rate of propagation reduced with the increasing distance (B); pre-SD baseline (Panel C) as well as the peak of hyperaemic flow response (Panel D) increased significantly. Data are given as mean \pm stdev. Analysis of variance (ANOVA) was used for statistical analysis $(* \mathrm{P}<0.05$ given as level of confidence).

Flow distribution was irregular, heterogeneous due to the unpredictable pattern of microsphere capture in vessels. However, blood flow in pial arterioles blocked by microspheres dropped immediately, with remaining high-flow rates in nearby arterioles due to reactive hyperemia. This sudden, inhomogeneous blood flow distribution between low and high flow areas leads to swelling of the brain [61] and may also contributes to further progression of injury. Whereas $\mathrm{CBF}$ reduction was immediate, the mean $\mathrm{CBF}$ drop in the field of view after embolization was not severe (CBF in $90 \%$ of the visible 
cortex stayed below 50\%) compared to global forebrain ischemia, where CBF decrease was homogenous, and more profound ( 40\%) after ischemia completion.

\subsection{Threshold of ischemia induced depolarization waves}

There is no evidence that SDs occur spontaneously in the intact cerebral cortex. Our results also confirm this notion, since no SD developed prior to ischemia induction. Moreover, in our global forebrain ischemia model, no SD was detected between 2VO onset and the beginning of blood withdrawal, which indicates that $2 \mathrm{VO}$ alone did not cause ischemia severe enough to favor the elicitation of spontaneous SDs.

In our global forebrain ischemia study, only one SD emerged 16-17 min after 2VOduring or after the completion of hypovolemic hypotension, when CBF varied around $43.4 \pm 4.9 \%$. No further SDs were detected during the observation period.

In previous studies, SDs occurred promptly after ischemia onset: in a global ischemia model achieved by cardiac arrest, terminal depolarization occurred 1-2 min after ischemia induction [49, 62, 63]. Similarly, in focal ischemia created by MCAO repeated, transient waves of depolarization emerged within a few minutes after vascular occlusion [51, 64]. The elongated latency of depolarization development with respect to the onset of the ischemic insult in global forebrain ischemia as shown here is suggested to be the result of a gradual progression of ischemia, in contrast with a sudden insult (i.e. abrupt vascular occlusion). It is suggested that CBF compensation operates effectively during gradual peripheral vascular challenges, which delays the development of cerebral ischemia severe enough to induce SD [65].
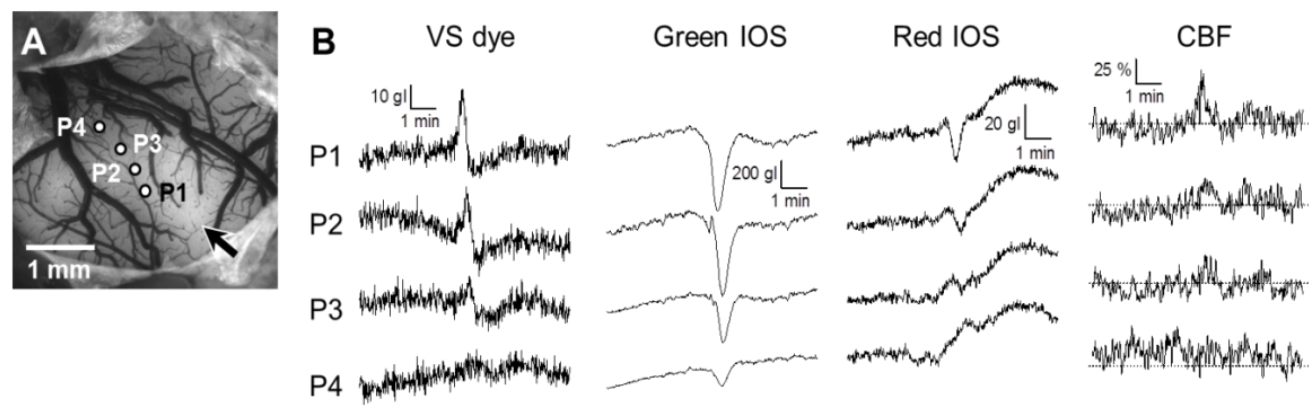

Figure 17: Spreading depolarization (SD) and the associated hemodynamic response gradually extinguished during propagation. Picture A shows the anatomy of the cortex under green illumination $(\lambda=540-550 \mathrm{~nm}$ ). Voltage sensitive (VS) dye emittance, green and red intrinsic optical signal (IOS), and cerebral blood flow (CBF) signals were acquired at selected areas of interest (P1-P4) perpendicular to the wave front. Kinetics of changes in VS dye signal, red and green IOS, and CBF are illustrated by P1-P4 traces. Direction of SD propagation is indicated by a black arrow (lower right corner of the cranial window) in Panel A. Intensities of VS dye fluorescence, and red and green IOS are given in gray level (gl). Dotted lines across the CBF traces indicate $60 \%$ of baseline. 
In contrast, multifocal ischemia gave rise to numerous (2-12) SDs over the hour following ischemia induction. The temporal distribution of these SDs showed a wide variation ( 3 to $37 \mathrm{~min}$ ) after ischemia induction, and the events emerged when CBF was of $56.9 \pm 9 \%$ (in visualized focus). Previously, the CBF threshold of SD elicitation was determined as $\sim 40 \mathrm{~mL} / 100 \mathrm{~g}$ per minute in the Spontaneously Hypertensive rat [66]. The finding that SDs generated at a CBF of approximately $43 \%$ in our global forebrain ischemia model in contrast with a considerably higher CBF of $57 \%$ in the multi-focal ischemia model indicates, that the level of perfusion deficit alone does not predict SD elicitation. We suggest that conditions other than the degree of perfusion deficit must also be involved; especially when ischemia is heterogeneous, sharp gradients of extracellular $\mathrm{K}^{+}$may promote $\mathrm{SD}$ elicitation, even in the face of relatively moderate local CBF reduction.

\subsection{The focal area of spreading depolarizations}

In global forebrain ischemia, the focus of SD elicitation fell out of the cortical region covered by the cranial window, and depolarization events propagated without attenuation. According to previous observations in global ischemia after cardiac arrest, depolarization in forebrain ischemia propagated from an unidentified origin that lies frontal to the site of observation (i.e. the parietal cortex) [49]. Frontolateral propagation suggested that SDs were generated either in the frontolateral cortex, or arrived at the cortex from deeper structures more sensitive to ischemia. The putative focus of SDs may be located in the border zone of the anterior and medial cerebral arteries, where blood supply is provided only by the collateral pial network of Heubner's anastomoses, which is highly sensitive to systemic blood pressure [67]. Alternatively, SD may originate in the striatum, and propagate to the cerebral cortex through an anterior route (i.e. claustrum and nucleus accumbens), as demonstrated earlier [64, 68, 69], since the striatum contains a mixed pool of cells with different susceptibility to ischemia compared to the cortex with relative uniform cytoarchytecture [70, 71]. In multifocal ischemia, the number of SDs recorded in each experiment and localization of SD focus showed quite some variation. The foci of five SDs were seen within the cortical area revealed by the cranial window, but the site of their origin was not a typical ischemic core region; most importantly because the tissue appeared to be viable as evidenced by its involvement in the propagation of subsequent SD waves. As reference, in MCAO models membrane depolarization appeared to be 
persistent in the core region (i.e. terminal depolarization evolved), and subsequent SDs circulated around the core, but never invaded it [26]. Our observations confirm, that the focus of SD does not necessary overlap with the ischemic core, as was also shown by other studies [72], and that SDs can emerge over mild ischemia [41]. Even though SD foci revealed in the present study were thus not associated with the immediate evolution of an evident ischemic core, it is possible that microinfarcts too small for detection with imaging were associated with SD events. It is also possible that non-terminal SD in ischemic tissue does not cause immediate injury; nonetheless, the reestablished membrane potential may not guarantee ultimate neuronal survival. Indeed, the impact of recurrent SDs that appear harmless alone can be additive together, and their increasing total duration corresponds with worse neurological outcome [20].

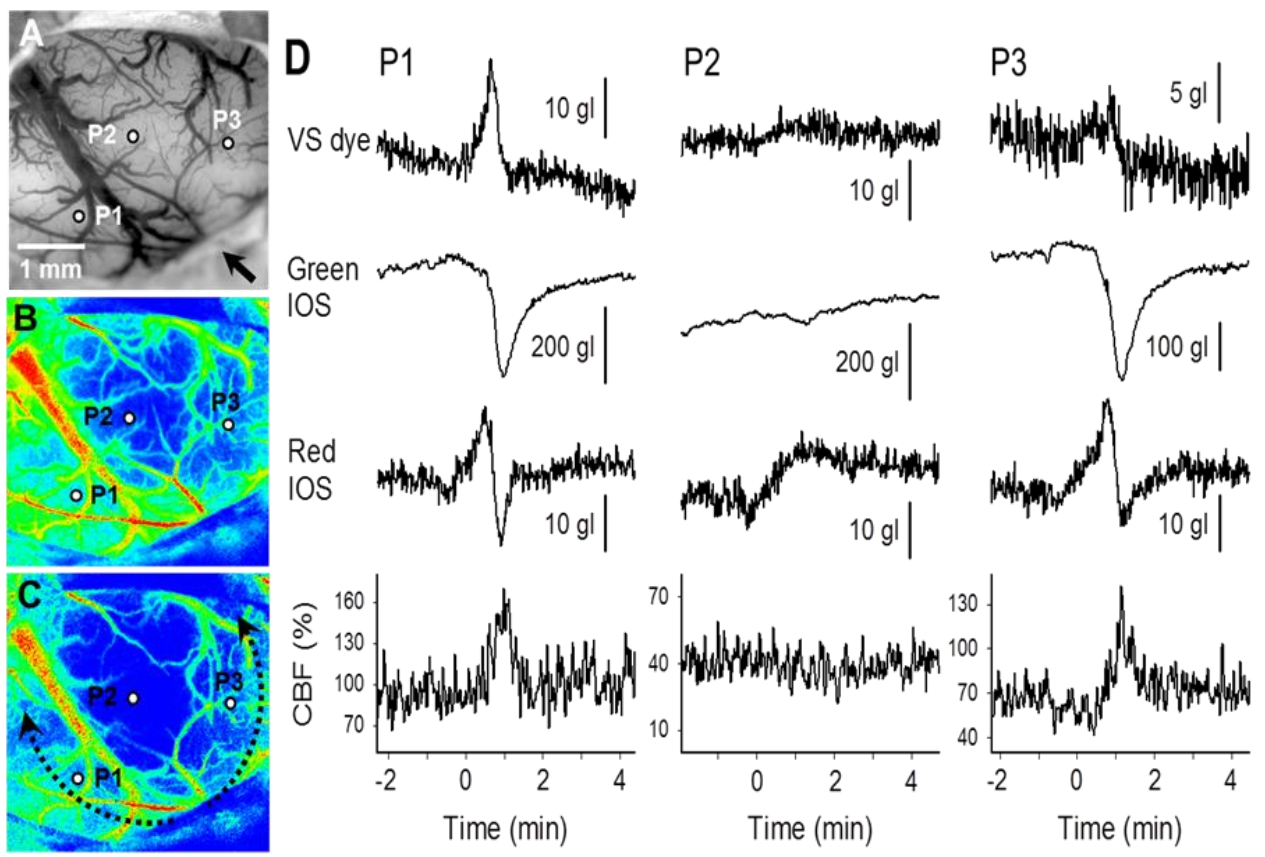

Figure 18: Spreading depolarization (SD) avoids cortical region with low perfusion. This particular SD invaded the cranial window from the frontolateral corner (Panel A, black arrow). In Panles B and C, pseudocolored cerebral blood flow (CBF) maps indicate low perfusion areas labeled with blue color. Panel B was captured during baseline, while Panel C represents ischemia. Arriving SD (dotted line arrows) did not invade the cortical area with low CBF. Representative traces taken at selected areas of interests (AOI P1-P3 in Panels B\&C) show voltage sensitive (VS) dye fluorescence, green and red intrinsic optical signal (IOS), and change of CBF relative to baseline. At P1-P3, where the drop of CBF after ischemia induction was milder, SD propagation was unhampered (peak in VS dye signal in Panel $\mathrm{D})$ and SD was coupled with hyperemic CBF response. In the central region (P2), where CBF drop was more prominent (to $\sim 40 \%$ respect to the baseline), no SD propagation was detected.

The present results also revealed that $\mathrm{CBF}$ before SD elicitation was the lowest, and the peak of SD-associated hyperemia was smallest at the focus of SDs, as compared with other sites more distant to the focus. These observations imply that in tissue with uniform 
structure (i.e., rodent cortex), SD is elicited at a site where the reduction in CBF reaches a critical threshold, and subsequently, neurovascular coupling is the least efficient to support functional hyperemia.

Here, we show that the duration of SD (time between depolarization and repolarization) tends to be prolonged at the proper focus compared with sampling sites more distant to the focus, and the rate of SD decelerates with the increasing distance from the focus. The rate of SD propagation is predicted to increase when (1) extracellular $\mathrm{K}^{+}$ removal is hampered, (2) the extracellular space is smaller (cell swelling), and (3) extracellular $\mathrm{K}^{+}$and/or glutamate concentrations are increased [73]. In line with the predictions, hypoxic SDs tended to propagate approximately $1 \mathrm{~mm} / \mathrm{min}$ more rapidly than normoxic SDs as observed in brain slices $[74,75]$. These data in combination with our findings suggest that the tissue at the SD focus may be more hypoxic than in the surrounding area.

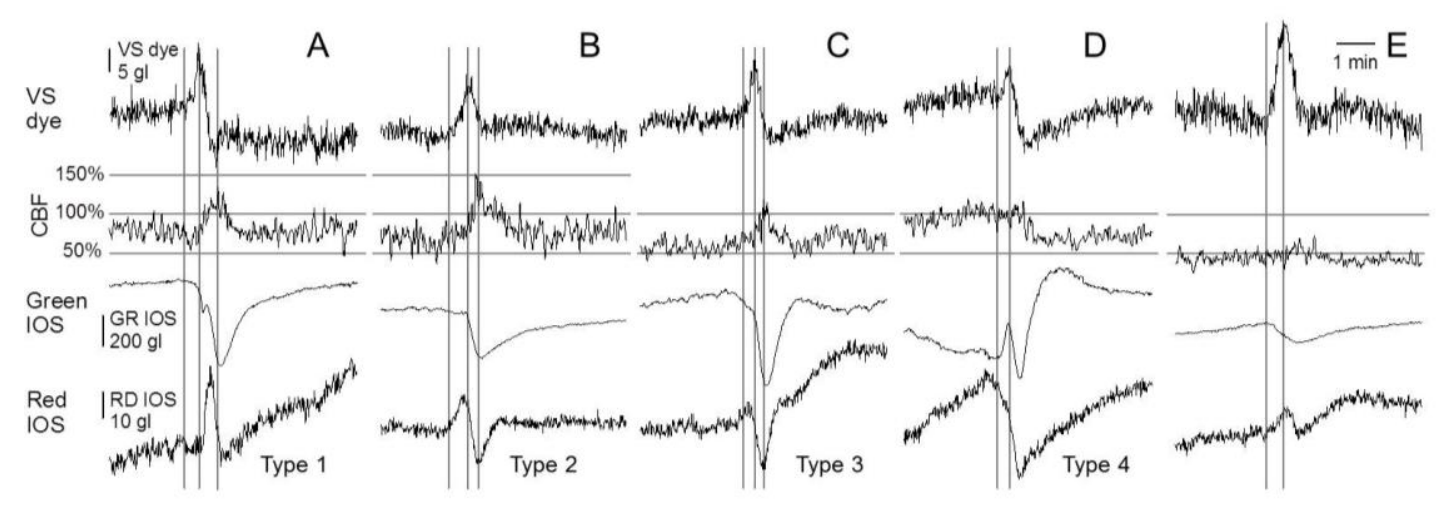

Figure 19: Spreading depolarization (SD) events, associated cerebral blood flow (CBF) transients, and intrinsic optical signal (IOS) changes. Representative traces show the kinetics of changes in voltage sensitive (VS) dye fluorescence intensity, local CBF, green IOS, and red IOS. Vertical gray guidelines help to appreciate the temporal relationship between the kinetics of corresponding optical signals. The guidelines are aligned to the start and peak of the SD related increase in VS dye fluorescence (A to E), and the peak of associated hyperemia where applicable (A to C). VS dye, red and green IOS changes are given in grey level (gl), while CBF in \%. Red IOS signatures with different kinetics are labeled as Type 1 to 4 (A to D).

\subsection{Kinetics of spreading depolarization and the associated cerebral blood flow response}

A major advantage of our experimental approach is that the kinetics of SD and the associated $\mathrm{CBF}$ response can be directly correlated. The duration of SD itself has been proposed to determine the duration of SD-related hyperemia, because longer duration of 
the DC shift coincided with longer hyperemia [76]. Since the recovery of resting membrane potential and thus normal electrical activity of the nervous tissue requires the activity of the ATP-consuming $\mathrm{Na}^{+}$pump [32], the return of CBF to baseline after hyperemia may be postponed by the continuing energy need, reflected by the longer duration of the electric silence with SD. On the other hand, the longer duration of the SDrelated spreading ischemia was suggested to delay repolarization, as nutrient supply necessary for repolarization does not match the need of the tissue [32].

In our global ischemia paradigm, repolarization after SD was either prolonged (prolonged type SDs), or no recovery of transmembrane potential was visualized (terminal SDs), and only few of the SD events proved to be short transient SDs. This is in agreement with clinical observations made in subarachnoid hemorrhage patients [19]. At the same time, none of the SDs were coupled with physiological, hyperemic flow response. Instead, prolonged and terminal SDs coincided with spreading ischemia with a duration overlapping with SD duration (Fig. 2), and no flow response was detected with the few short transient SDs.

In the ischemic brain, the $\mathrm{CBF}$ response to $\mathrm{SD}$ is more dominated by vasoconstrictive elements, leading to diminishing hyperemia and more prevalent hypoemia [33, 76, 77]. In the most severe form, the hypoemic element completely outweighs hyperemia, and turns into spreading ischemia [32]. Vasoconstriction may prevail because local vasoconstrictive mediation becomes accentuated or because counteracting vasodilators that would normally balance out vasoconstriction are not available. A key element of vasoregulation mediating the evolution of spreading ischemia was identified as perivascular $\mathrm{K}^{+}$ concentration, increasing above the dilation/constriction threshold (i.e. $20 \mathrm{mM}$ ) [39]. In addition, excessive extracellular $\mathrm{K}^{+}$was suggested to accumulate in response to electrical field stimulation after experimental subarachnoid hemorrhage as the result of efflux from astrocytes via large-conductance $\mathrm{Ca} 2+-$ activated $\mathrm{K}+$ channels. Finally, NO scavenging (e.g. by hemoglobin) has been proposed as a potent mechanism to limit vasodilator NO availability, thereby creating vasoconstrictive CBF response to SD [35].

In summary, delayed repolarization or longer SD duration, as well as the insufficiency of the associated CBF response are clear signs of energy crisis in the tissue, and reflect the inability of ion pumps to re-establish resting membrane potential, as was demonstrated in patients and animal models of cerebral ischemia [20, 21, 78]. Moreover, persistent depolarization (i.e. no recovery of transmembrane potential) or continuing 
spreading ischemia indicate irreversible energy crisis and predicts consequent neuronal damage.

In our multifocal ischemia model, SDs were short transient, and the SD related CBF response was invariably hyperemic, albeit the hypoemic elements of the response (i.e. initial brief hypoperfusion and prolonged oligemia) remained undetectable. We speculate that both the initial, brief decrease in CBF and the final prolonged oligemic component remained obscure during ischemia, because $\mathrm{CBF}$ shortly before $\mathrm{SD}$ occurrence was already low due to the created perfusion deficit (Fig. 2B). The diverse kinetics of changes in red IOS intensity (representative of $\mathrm{Hb}$ saturation) with transient hyperemia (Fig. 18) denotes various metabolic consequences of SDs in the ischemic cortex. Because the Type 1 to 3 red IOS intensity changes were all coupled to transient hyperemia, and the types of red IOS signature were unrelated to the magnitude of hyperemia, the analysis of the kinetics of hyperemic CBF responses alone appears not to be sufficient to draw conclusions about the metabolic consequences of SD. Our future studies have been designed to unravel the metabolic burden associated with hyperemic CBF responses to SDs propagating over the ischemic cortex.

\subsection{Unique features of spreading depolarizations over their course of propagation}

The multifocal ischemia study provided an excellent opportunity to follow SDs along their course of propagation over tissue of heterogeneous metabolic status. We observed that some propagating SDs gradually diminished over their course of traveling. Such diminishing waves were previously seen in the chicken retina ex vivo [79]. Moreover, the hyperemic response to SD was seen to die away in stroke patients [77]. We propose that a diminishing SD may propagate against an extracellular ionic gradient that does not support depolarization to evolve (possibly decreasing $\mathrm{K}^{+}$levels as a result of enhanced reabsorption, or increasingly higher $\mathrm{Mg}^{2+}$ concentrations), and brings the SD to a gradual halt. Alternatively, hampered water movement through glial aquaporin channels was proposed to counteract the propagation of SD (Fernandes de Lima et al., 2011), which may be another potential mechanism to bring SD to cessation.

A few SDs in our experiments avoided a distinct region of the visible cortex. In contrast with the diminishing waves presented above, these SDs were not extinguished gradually, but sharply curved around an area showing the lowest CBF in the field of view, and thus propagated with an irregular wave front. Similar to these observations, LSC 
imaging identified circumferential propagation of SD-related flow responses around areas defined as primary ischemic core regions in rats and cats [26]. According to the best of our knowledge, an SD does not invade a bulk of tissue if (i) the area has depolarized terminally (therefore subsequent depolarization cannot occur) or (ii) the area is suffering of ongoing epileptic activity [80]. Primary infarct evolution in the area avoided by SDs was not seen in our experiments (i.e., signature of potential terminal depolarization was not acquired), therefore infarct maturation cannot be responsible for the obstruction of SD propagation. Focal seizures are known as potential consequences of ischemic brain injury [64], and microembolization in the rat has been shown to induce seizures [81], but the methods applied here did not allow seizure detection to justify or exclude seizure activity. All things considered, presently we can only claim that the area not being able to substantiate SD propagation was of the lowest perfusion within the field of view, but was presumably not infarcted.

\section{Conclusions and future perspectives}

Simultaneous imaging of changes in membrane potential and hemodynamic variables during global forebrain ischemia revealed, that sustained and transient hypoperfusion occurred in response to persistent and intermediate depolarization, respectively, whereas short, transient depolarization produced no clear flow response. Under less severe perfusion deficit produced in a multi-focal cerebral ischemia model, hyperemic CBF responses were coupled to short, transient SDs. In addition, hyperemic responses to SDs were shown to be associated with various kinetics of $\mathrm{Hb}$ saturation during ischemia, despite the similar amplitude and duration of hyperemia. Accepting that inverse coupling (e.g. hypoperfusion evolving in response to SD) jeopardizes the integrity of the nervous tissue [32], hyperemic CBF responses alone may have low predictive value as to the metabolic crisis during SD propagation in the tissue. In order to evaluate the metabolic impact of SDs with hyperemic CBF responses, hemoglobin saturation, tissue lactate level or tissue $\mathrm{pH}$ must be assessed.

The results of the global forebrain ischemia experiments may be relevant to human conditions such as hypoxic-ischemic brain injury as a consequence of cardiac arrest or hypoxia during surgery or critical illness. At the same time, microsphere-induced recurrent SDs in the rat cerebral cortex indicate that SDs could evolve in small embolic infarcts in patients, complications with cardiac or pulmonary arteriovenous shunts (e.g., 
cerebral emboli), and possibly multi-infarct dementia. The potential pathogenic role of SDs in these conditions is still to be clarified. 


\section{References}

1.Leao, A.A., Spreading depression of activity in the cerebral cortex. J Neurophysiol, 1944(7): p. 359-390.

2.Hartings, J.A., et al., The continuum of spreading depolarizations in acute cortical lesion development: Examining Leao's legacy. J Cereb Blood Flow Metab, 2016.

3.Dreier, J.P., et al., Recording, analysis, and interpretation of spreading depolarizations in neurointensive care: Review and recommendations of the COSBID research group. J Cereb Blood Flow Metab, 2016.

4.Nicholson, C., et al., Potassium, calcium, chloride and sodium changes in extracellular space during spreading depression in cerebellum [proceedings].

Arzneimittelforschung, 1978. 28(5): p. 874-5.

5.Hansen, A.J. and T. Zeuthen, Extracellular ion concentrations during spreading depression and ischemia in the rat brain cortex. Acta Physiol Scand, 1981. 113(4): p. $437-45$.

6.Somjen, G.G., Mechanisms of spreading depression and hypoxic spreading depressionlike depolarization. Physiol Rev, 2001. 81(3): p. 1065-96.

7.Takano, T., et al., Cortical spreading depression causes and coincides with tissue hypoxia. Nat Neurosci, 2007. 10(6): p. 754-62.

8.Risher, W.C., D. Croom, and S.A. Kirov, Persistent astroglial swelling accompanies rapid reversible dendritic injury during stroke-induced spreading depolarizations. Glia, 2012. 60(11): p. 1709-20.

9.Herreras, O., et al., Role of neuronal synchronizing mechanisms in the propagation of spreading depression in the in vivo hippocampus. J Neurosci, 1994. 14(11 Pt 2): p. 7087-98.

10.Rossi, D.J., T. Oshima, and D. Attwell, Glutamate release in severe brain ischaemia is mainly by reversed uptake. Nature, 2000. 403(6767): p. 316-21.

11.Zonta, M., et al., Neuron-to-astrocyte signaling is central to the dynamic control of brain microcirculation. Nat Neurosci, 2003. 6(1): p. 43-50.

12.Cauli, B. and E. Hamel, Revisiting the role of neurons in neurovascular coupling. Front Neuroenergetics, 2010. 2: p. 9.

13.Attwell, D., et al., Glial and neuronal control of brain blood flow. Nature, 2010. 468(7321): p. 232-43.

14.Ayata, C. and M. Lauritzen, Spreading Depression, Spreading Depolarizations, and 
the Cerebral Vasculature. Physiol Rev, 2015. 95(3): p. 953-93.

15.Lauritzen, M., Pathophysiology of the migraine aura. The spreading depression theory. Brain, 1994. 117 ( Pt 1): p. 199-210.

16.Leao, A.A., Further observations on the spreading depression of activity in the cerebral cortex. J Neurophysiol, 1947. 10(6): p. 409-14.

17.Strong, A.J., et al., Spreading and synchronous depressions of cortical activity in acutely injured human brain. Stroke, 2002. 33(12): p. 2738-43.

18.Dohmen, C., et al., Spreading depolarizations occur in human ischemic stroke with high incidence. Ann Neurol, 2008. 63(6): p. 720-8.

19.Dreier, J.P., et al., Cortical spreading ischaemia is a novel process involved in ischaemic damage in patients with aneurysmal subarachnoid haemorrhage. Brain, 2009. 132(Pt 7): p. 1866-81.

20.Dreier, J.P., et al., Delayed ischaemic neurological deficits after subarachnoid haemorrhage are associated with clusters of spreading depolarizations. Brain, 2006. 129(Pt 12): p. 3224-37.

21.Fabricius, M., et al., Cortical spreading depression and peri-infarct depolarization in acutely injured human cerebral cortex. Brain, 2006. 129(Pt 3): p. 778-90.

22.Hartings, J.A., et al., Spreading depolarisations and outcome after traumatic brain injury: a prospective observational study. Lancet Neurol, 2011. 10(12): p. 1058-64.

23.Hartings, J.A., et al., Spreading depolarizations have prolonged direct current shifts and are associated with poor outcome in brain trauma. Brain, 2011. 134(Pt 5): p. 1529-40.

24.Busch, E., et al., Potassium-induced cortical spreading depressions during focal cerebral ischemia in rats: contribution to lesion growth assessed by diffusion-weighted NMR and biochemical imaging. J Cereb Blood Flow Metab, 1996. 16(6): p. 1090-9.

25.Hossmann, K.A., Viability thresholds and the penumbra of focal ischemia. Ann Neurol, 1994. 36(4): p. 557-65.

26.Nakamura, H., et al., Spreading depolarizations cycle around and enlarge focal ischaemic brain lesions. Brain, 2010. 133(Pt 7): p. 1994-2006.

27.Mies, G., T. Iijima, and K.A. Hossmann, Correlation between peri-infarct DC shifts and ischaemic neuronal damage in rat. Neuroreport, 1993. 4(6): p. 709-11.

28.Dijkhuizen, R.M., et al., Correlation between tissue depolarizations and damage in focal ischemic rat brain. Brain Res, 1999. 840(1-2): p. 194-205.

29.Takano, K., et al., The role of spreading depression in focal ischemia evaluated by 
diffusion mapping. Ann Neurol, 1996. 39(3): p. 308-18.

30.Nedergaard, M. and A.J. Hansen, Spreading depression is not associated with neuronal injury in the normal brain. Brain Res, 1988. 449(1-2): p. 395-8.

31.Oliveira-Ferreira, A.I., et al., Experimental and preliminary clinical evidence of an ischemic zone with prolonged negative DC shifts surrounded by a normally perfused tissue belt with persistent electrocorticographic depression. J Cereb Blood Flow Metab, 2010. 30(8): p. 1504-19.

32.Dreier, J.P., The role of spreading depression, spreading depolarization and spreading ischemia in neurological disease. Nat Med, 2011. 17(4): p. 439-47.

33. Hoffmann, U., et al., Calabadion: A new agent to reverse the effects of benzylisoquinoline and steroidal neuromuscular-blocking agents. Anesthesiology, 2013. 119(2): p. 317-25.

34.Bere, Z., et al., Imaging reveals the focal area of spreading depolarizations and a variety of hemodynamic responses in a rat microembolic stroke model. J Cereb Blood Flow Metab, 2014. 34(10): p. 1695-705.

35.Dreier, J.P., et al., Nitric oxide scavenging by hemoglobin or nitric oxide synthase inhibition by $N$-nitro-L-arginine induces cortical spreading ischemia when $\mathrm{K}+\mathrm{is}$ increased in the subarachnoid space. J Cereb Blood Flow Metab, 1998. 18(9): p. 97890.

36.Iadecola, C., et al., Nitric oxide synthase inhibition and cerebrovascular regulation. J Cereb Blood Flow Metab, 1994. 14(2): p. 175-92.

37.Golding, E.M., et al., Potentiated endothelium-derived hyperpolarizing factormediated dilations in cerebral arteries following mild head injury. J Neurotrauma, 2001. 18(7): p. 691-7.

38.Macdonald, R.L., Delayed neurological deterioration after subarachnoid haemorrhage. Nat Rev Neurol, 2014. 10(1): p. 44-58.

39.Windmuller, O., et al., Ion changes in spreading ischaemia induce rat middle cerebral artery constriction in the absence of NO. Brain, 2005. 128(Pt 9): p. 2042-51.

40.Mulligan, S.J. and B.A. MacVicar, Calcium transients in astrocyte endfeet cause cerebrovascular constrictions. Nature, 2004. 431(7005): p. 195-9.

41.Dreier, J.P., et al., Ischemia triggered by red blood cell products in the subarachnoid space is inhibited by nimodipine administration or moderate volume expansion/hemodilution in rats. Neurosurgery, 2002. 51(6): p. 1457-65; discussion 1465-7. 
42.Sukhotinsky, I., et al., Hypoxia and hypotension transform the blood flow response to cortical spreading depression from hyperemia into hypoperfusion in the rat. $\mathrm{J}$ Cereb Blood Flow Metab, 2008. 28(7): p. 1369-76.

43.Shin, H.K., et al., Vasoconstrictive neurovascular coupling during focal ischemic depolarizations. J Cereb Blood Flow Metab, 2006. 26(8): p. 1018-30.

44.Strong, A.J., J.A. Hartings, and J.P. Dreier, Cortical spreading depression: an adverse but treatable factor in intensive care? Curr Opin Crit Care, 2007. 13(2): p. 126-33.

45.Farkas, E., et al., Direct, live imaging of cortical spreading depression and anoxic depolarisation using a fluorescent, voltage-sensitive dye. J Cereb Blood Flow Metab, 2008. 28(2): p. 251-62.

46. Grinvald, A. and R. Hildesheim, VSDI: a new era in functional imaging of cortical dynamics. Nat Rev Neurosci, 2004. 5(11): p. 874-85.

47.Obrenovitch, T.P., S. Chen, and E. Farkas, Simultaneous, live imaging of cortical spreading depression and associated cerebral blood flow changes, by combining voltage-sensitive dye and laser speckle contrast methods. Neuroimage, 2009. 45(1): p. 68-74.

48.Ayata, C., et al., Laser speckle flowmetry for the study of cerebrovascular physiology in normal and ischemic mouse cortex. J Cereb Blood Flow Metab, 2004. 24(7): p. 74455 .

49.Farkas, E., F. Bari, and T.P. Obrenovitch, Multi-modal imaging of anoxic depolarization and hemodynamic changes induced by cardiac arrest in the rat cerebral cortex. Neuroimage, 2010. 51(2): p. 734-42.

50.Traystman, R.J., Animal models of focal and global cerebral ischemia. Ilar j, 2003. 44(2): p. 85-95.

51.Hossmann, K.A., Periinfarct depolarizations. Cerebrovasc Brain Metab Rev, 1996. 8(3): p. 195-208.

52.Miyake, K., S. Takeo, and H. Kaijihara, Sustained decrease in brain regional blood flow after microsphere embolism in rats. Stroke, 1993. 24(3): p. 415-20.

53.Mayzel-Oreg, O., et al., Microsphere-induced embolic stroke: an MRI study. Magn Reson Med, 2004. 51(6): p. 1232-8.

54.Nozari, A., et al., Microemboli may link spreading depression, migraine aura, and patent foramen ovale. Ann Neurol, 2010. 67(2): p. 221-9.

55.Dahlem, Y.A. and W. Hanke, Intrinsic optical signal of retinal spreading depression: second phase depends on energy metabolism and nitric oxide. Brain Res, 2005. 
1049(1): p. 15-24.

56.Hartings, J.A., et al., Spreading depolarizations and late secondary insults after traumatic brain injury. J Neurotrauma, 2009. 26(11): p. 1857-66.

57.de Castro, M.T., J.C. de Castro, and J.V. Menani, The effects of forebrain multiple lesions on the pressor response induced by bilateral carotid occlusion in conscious rats. Brain Res, 1993. 612(1-2): p. 243-6.

58.Faris, I.B., G.G. Jamieson, and J. Ludbrook, Effects of acute changes in blood volume on the carotid sinus baroreceptor reflex in conscious rabbits. J Physiol, 1983. 337: p. $563-73$.

59.Choy, M., et al., The chronic vascular and haemodynamic response after permanent bilateral common carotid occlusion in newborn and adult rats. J Cereb Blood Flow Metab, 2006. 26(8): p. 1066-75.

60.Wiernsperger, N., A.L. Sylvia, and F.F. Jobsis, Incomplete transient ischemia: a nondestructive evaluation of in vivo cerebral metabolism and hemodynamics in rat brain. Stroke, 1981. 12(6): p. 864-8.

61.Hossmann, K.A., Reperfusion of the brain after global ischemia: hemodynamic disturbances. Shock, 1997. 8(2): p. 95-101; discussion 102-3.

62.Xie, Y., et al., Ion channel involvement in anoxic depolarization induced by cardiac arrest in rat brain. J Cereb Blood Flow Metab, 1995. 15(4): p. 587-94.

63.de Crespigny, A.J., et al., Rapid monitoring of diffusion, DC potential, and blood oxygenation changes during global ischemia. Effects of hypoglycemia, hyperglycemia, and TTX. Stroke, 1999. 30(10): p. 2212-22.

64.Hartings, J.A., et al., Delayed secondary phase of peri-infarct depolarizations after focal cerebral ischemia: relation to infarct growth and neuroprotection. J Neurosci, 2003. 23(37): p. 11602-10.

65.Nakase, H., et al., Cerebral blood flow and tissue oxygen saturation in immediate and progressive ischemia in rat brain. Neurol Res, 2001. 23(8): p. 875-80.

66.Takeda, Y., et al., Metabolic and perfusion responses to recurrent peri-infarct depolarization during focal ischemia in the Spontaneously Hypertensive Rat: dominant contribution of sporadic CBF decrements to infarct expansion. J Cereb Blood Flow Metab, 2011. 31(9): p. 1863-73.

67.Henninger, N., A. Heimann, and O. Kempski, Electrophysiology and neuronal integrity following systemic arterial hypotension in a rat model of unilateral carotid artery occlusion. Brain Res, 2007. 1163: p. 119-29. 
68.Umegaki, M., et al., Peri-infarct depolarizations reveal penumbra-like conditions in striatum. J Neurosci, 2005. 25(6): p. 1387-94.

69.Vinogradova, L.V., V.I. Koroleva, and J. Bures, Re-entry waves of Leao's spreading depression between neocortex and caudate nucleus. Brain Res, 1991. 538(1): p. 161-4.

70.Chesselet, M.F., et al., Ischemic damage in the striatum of adult gerbils: relative sparing of somatostatinergic and cholinergic interneurons contrasts with loss of efferent neurons. Exp Neurol, 1990. 110(2): p. 209-18.

71.Pulsinelli, W.A., Selective neuronal vulnerability: morphological and molecular characteristics. Prog Brain Res, 1985. 63: p. 29-37.

72.Rother, J., et al., MR detection of cortical spreading depression immediately after focal ischemia in the rat. J Cereb Blood Flow Metab, 1996. 16(2): p. 214-20.

73.Zandt, B.J., B. ten Haken, and M.J. van Putten, Diffusing substances during spreading depolarization: analytical expressions for propagation speed, triggering, and concentration time courses. J Neurosci, 2013. 33(14): p. 5915-23.

74.Aitken, P.G., et al., Similar propagation of SD and hypoxic SD-like depolarization in rat hippocampus recorded optically and electrically. J Neurophysiol, 1998. 80(3): p. 1514-21.

75.Joshi, I. and R.D. Andrew, Imaging anoxic depolarization during ischemia-like conditions in the mouse hemi-brain slice. J Neurophysiol, 2001. 85(1): p. 414-24.

76.Menyhart, A., et al., High incidence of adverse cerebral blood flow responses to spreading depolarization in the aged ischemic rat brain. Neurobiol Aging, 2015. 36(12): p. 3269-77.

77.Woitzik, J., et al., Propagation of cortical spreading depolarization in the human cortex after malignant stroke. Neurology, 2013. 80(12): p. 1095-102.

78.Nedergaard, M. and A.J. Hansen, Characterization of cortical depolarizations evoked in focal cerebral ischemia. J Cereb Blood Flow Metab, 1993. 13(4): p. 568-74.

79.Martins-Ferreira, H., M. Nedergaard, and C. Nicholson, Perspectives on spreading depression. Brain Res Brain Res Rev, 2000. 32(1): p. 215-34.

80.Koroleva, V.I. and J. Bures, Stimulation induced recurrent epileptiform discharges block cortical and subcortical spreading depression in rats. Physiol Bohemoslov, 1982. 31(5): p. 385-400.

81.Tsai, M.J., Y.H. Tsai, and Y.M. Kuo, Characterization of the pattern of ischemic stroke induced by artificial particle embolization in the rat brain. Biomaterials, 2011. 32(27): p. 6381-8. 